\title{
Astrochemical Properties of Planck Cold Clumps
}

Ken'ichi Tatematsu ${ }^{1,2}$, Tie Liu ${ }^{3}$, Satoshi Ohashi ${ }^{4}$, Patricio Sanhueza ${ }^{1}$, Quang Nguyễn Lu'o'ng ${ }^{1,3}$, Tomoya Hirota ${ }^{1,2}$, Sheng-Yuan Liu ${ }^{5}$, Naomi Hirano, ${ }^{5}$, Minho $\mathrm{Choi}^{3}$, Miju Kang ${ }^{3}$, Mark A.Thompson ${ }^{6}$, Gary Fuller ${ }^{7}$, Yuefang $\mathrm{Wu}^{8}, \mathrm{Di} \mathrm{Li}^{9}$, James Di Francesco $^{10,11}$, Kee-Tae Kim ${ }^{3}$, Ke Wang ${ }^{12}$, Isabelle Ristorcelli ${ }^{13}$, Mika Juvela ${ }^{14}$, Hiroko Shinnaga ${ }^{15}$, Maria Cunningham ${ }^{16}$, Masao Saito ${ }^{17}$, Jeong-Eun Lee ${ }^{18}$, L. Viktor Tóth ${ }^{19}$, Jinhua He ${ }^{20,21,22}$, Takeshi Sakai ${ }^{23}$, Jungha Kim ${ }^{18}$ (JCMT Large Program "SCOPE" collaboration) and

(TRAO Key Science Program “TOP” collaboration)

\footnotetext{
${ }^{1}$ National Astronomical Observatory of Japan, National Institutes of Natural Sciences, 2-21-1 Osawa, Mitaka, Tokyo 181-8588, Japan; k.tatematsu@nao.ac.jp

${ }^{2}$ Department of Astronomical Science, SOKENDAI (The Graduate University for Advanced Studies), 2-21-1 Osawa, Mitaka, Tokyo 181-8588, Japan

${ }^{3}$ Korea Astronomy and Space Science Institute, Daedeokdaero 776, Yuseong, Daejeon 305-348, Korea

${ }^{4}$ Department of Astronomy, The University of Tokyo, Bunkyo-ku, Tokyo 113-0033, Japan

${ }^{5}$ Academia Sinica Institute of Astronomy and Astrophysics, 11F of Astronomy-Mathematics Building, AS/NTU. No.1, Section 4, Roosevelt Rd, Taipei 10617, Taiwan, R.O.C.

${ }^{6}$ Centre for Astrophysics Research, Science \& Technology Research Institute, University of Hertfordshire, Hatfield, AL10 9AB, UK

${ }^{7}$ Jodrell Bank Centre for Astrophysics, School of Physics and Astronomy, University of Manchester, Oxford Road, Manchester, M13 9PL, UK ${ }^{8}$ Department of Astronomy, Peking University, 100871, Beijing, China

${ }^{9}$ National Astronomical Observatories, Chinese Academy of Sciences, Beijing, 100012, China

${ }^{10}$ NRC Herzberg Astronomy and Astrophysics, 5071 West Saanich Road, Victoria, BC V9E 2E7, Canada

${ }^{11}$ Department of Physics and Astronomy, University of Victoria, Victoria, BC V8P 1A1, Canada

${ }^{13}$ IRAP, CNRS (UMR5277), Universite Paul Sabatier, 9 avenue du Colonel Roche, BP 44346, F-31028, Toulouse Cedex 4, France

${ }^{14}$ Department of physics, University of Helsinki, FI-00014, Helsinki, Finland

${ }^{15}$ Department of Physics, Kagoshima University, 1-21-35, Korimoto, Kagoshima, 890-0065, Japan

${ }^{16}$ School of Physics, University of New South Wales, Sydney, NSW 2052, Australia

${ }^{17}$ Nobeyama Radio Observatory, National Astronomical Observatory of Japan, National Institutes of Natural Sciences, Nobeyama, Minamimaki, Minamisaku, Nagano 384-1305, Japan

${ }^{18}$ School of Space Research, Kyung Hee University, Seocheon-Dong, Giheung-Gu, Yongin-Si, Gyeonggi-Do, 446-701, Korea

19 Department of Astronomy, Eötvös Loránd Unviersity, Pázmany Péter sétny 1, 1117 Budapest, Hungary

${ }^{20}$ Key Laboratory for the Structure and Evolution of Celestial Objects, Yunnan Observatories, Chinese Academy of Sciences, P.O. Box 110, Kunming, 650011, Yunnan Province, China

${ }^{21}$ Chinese Academy of Sciences, South America Center for Astrophysics (CASSACA), Camino El Observatorio 1515, Las Condes, Santiago, Chile

${ }^{22}$ Departamento de Astronomía, Universidad de Chile, Casilla 36-D, Santiago, Chile

${ }^{23}$ Graduate School of Informatics and Engineering, The University of Electro-Communications, Chofu, Tokyo 182-8585, Japan Received 2016 October 11; revised 2016 November 30; accepted 2016 December 1; published 2017 March 1
}

\begin{abstract}
We observed 13 Planck cold clumps with the James Clerk Maxwell Telescope/SCUBA-2 and with the Nobeyama $45 \mathrm{~m}$ radio telescope. The $\mathrm{N}_{2} \mathrm{H}^{+}$distribution obtained with the Nobeyama telescope is quite similar to SCUBA-2 dust distribution. The $82 \mathrm{GHz} \mathrm{HC}_{3} \mathrm{~N}, 82 \mathrm{GHz} \mathrm{CCS}$, and $94 \mathrm{GHz} \mathrm{CCS}$ emission are often distributed differently with respect to the $\mathrm{N}_{2} \mathrm{H}^{+}$emission. The CCS emission, which is known to be abundant in starless molecular cloud cores, is often very clumpy in the observed targets. We made deep single-pointing observations in DNC, $\mathrm{HN}^{13} \mathrm{C}, \mathrm{N}_{2} \mathrm{D}^{+}$, and cyclic- $\mathrm{C}_{3} \mathrm{H}_{2}$ toward nine clumps. The detection rate of $\mathrm{N}_{2} \mathrm{D}^{+}$is $50 \%$. Furthermore, we observed the $\mathrm{NH}_{3}$ emission toward 15 Planck cold clumps to estimate the kinetic temperature, and confirmed that most targets are cold $(\lesssim 20 \mathrm{~K})$. In two of the starless clumps we observed, the CCS emission is distributed as it surrounds the $\mathrm{N}_{2} \mathrm{H}^{+}$core (chemically evolved gas), which resembles the case of L1544, a prestellar core showing collapse. In addition, we detected both DNC and $\mathrm{N}_{2} \mathrm{D}^{+}$. These two clumps are most likely on the verge of star formation. We introduce the chemical evolution factor (CEF) for starless cores to describe the chemical evolutionary stage, and analyze the observed Planck cold clumps.
\end{abstract}

Key words: ISM: clouds - ISM: molecules - ISM: structure - stars: formation

\section{Introduction}

On the basis of the Planck all-sky survey (Planck Collaboration XXIII 2011; Planck Collaboration XXVIII 2016), we are carrying out a series of observations of molecular clouds as the Planck cold clump collaboration in order to understand the initial condition for star formation (Liu et al. 2015). Planck cold clumps have low dust temperatures (10-20 $\mathrm{K}$; median $=14.5 \mathrm{~K})$. Pilot observations have been carried out with various ground-based telescopes such as the James Clerk Maxwell Telescope (JCMT), IRAM, PMO $14 \mathrm{~m}$, APEX, Mopra, Effelsberg, CSO, and SMA (Liu et al. 2015). A Large Program for JCMT dust continuum observations with SCUBA2 (SCOPE $^{24}$ : SCUBA-2 Continuum Observations of Preprotostellar Evolution; M. A. Thompson et al. 2017, in

\footnotetext{
${ }^{24}$ https://www.eaobservatory.org/jcmt/science/large-programs/scope/
} 
preparation) and a Key Science Program with TRAO $14 \mathrm{~m}$ radio telescope $\left(\mathrm{TOP}^{25}\right.$ : TRAO Observations of Planck cold clumps; T. Liu et al. 2017, in preparation) are ongoing.

To characterize Planck cold clumps, it is essential to investigate their chemical and physical properties in detail. In particular, we try to make their evolutionary stages clear. The chemical evolution of molecular clouds has been established to some extent, not only for nearby dark clouds (e.g., Suzuki et al. 1992; Hirahara et al. 1992; Benson et al. 1998; Hirota \& Yamamoto 2006; Hirota et al. 2009), but also for giant molecular clouds (GMCs; Tatematsu et al. 2010, 2014b; Ohashi et al. 2014 for Orion A GMC; Ohashi et al. 2016a for Vela C GMC; Sanhueza et al. 2012; Hoq et al. 2013 for infrared dark clouds). Carbon-chain molecules such as $\mathrm{CCS}$ and $\mathrm{HC}_{3} \mathrm{~N}$ tend to be abundant in starless molecular cloud cores, while $\mathrm{N}$-bearing molecules such as $\mathrm{NH}_{3}$ and $\mathrm{N}_{2} \mathrm{H}^{+}$as well as cyclic- $\mathrm{C}_{3} \mathrm{H}_{2}$ (c- $\left.\mathrm{C}_{3} \mathrm{H}_{2}\right)$ tend to be abundant in star-forming molecular cloud cores. However, $\mathrm{N}_{2} \mathrm{H}^{+}$will be destroyed by evaporated $\mathrm{CO}$ in warm cores having $T_{\text {dust }} \gtrsim 25 \mathrm{~K}$ (Lee et al. 2004), and therefore $N\left(\mathrm{~N}_{2} \mathrm{H}^{+}\right) / N(\mathrm{CCS})$ may not be a good evolutionary tracer for $T_{\text {dust }} \gtrsim 25 \mathrm{~K}$. In a survey of nearby cold dark cloud cores, Suzuki et al. (1992) detected CCS at $45 \mathrm{GHz}$ in $55 \%$ of the observed cores, and found that CCS is a tracer of the young molecular gas in starless cloud cores. Tatematsu et al. (2010) detected $\operatorname{CCSJ}_{N}=4_{3}-3_{2}$ at $45 \mathrm{GHz}$ in $32 \%$ of the cores observed toward Orion A GMC. Sakai et al. (2008) observed 55 massive clumps associated with infrared dark clouds (IRDCs) in CCS $J_{N}=4_{3}-3_{2}$ at $45 \mathrm{GHz}$ using the Nobeyama $45 \mathrm{~m}$ telescope, and detected this line in none of them. Dirienzo et al. (2015) observed nine IRDCs in CCS $J_{N}=2_{1}-1_{0}$ at $24 \mathrm{GHz}$ using VLA, and detected this line in all of them.

Furthermore, deuterium fractionation ratios are powerful evolutionary tracers (Hirota \& Yamamoto 2006; Sakai et al. 2012). The deuterium fraction $\mathrm{D} / \mathrm{H}$ in molecules in molecular clouds is larger than the terrestrial abundance ratio $\left(1.15 \times 10^{-4}\right)$ or the protosolar estimate $\left(2 \times 10^{-5}\right)$ (Geiss $\&$ Reeves 1981). The deuterium fraction is enhanced in molecular cloud cores before star formation (prestellar phase), which is cold enough, and the ratio increases with prestellar evolution until star formation (Crapsi et al. 2005; Hirota \& Yamamoto 2006). However, it should be noted that the deuterium fraction decreases with increasing temperature (Snell \& Wootten 1979; Wootten 1987; Schilke et al. 1992; Tatematsu et al. 2010). After star formation, the deuterium fraction decreases (Emprechtinger et al. 2009). $N(\mathrm{DNC}) / N$ (HNC) will decrease, which may in part be due to an increase in the kinetic temperature, but may be affected by a larger reaction timescale (several times $10^{4}$ years) while $N\left(\mathrm{~N}_{2} \mathrm{D}^{+}\right) / N$ $\left(\mathrm{N}_{2} \mathrm{H}^{+}\right)$will reflect the current temperature more directly, because of a shorter reaction timescale (less than 100 years) (Sakai et al. 2012).

We investigate the evolutionary stages of Planck cold clumps using molecular column density ratios. For this purpose, by using the Nobeyama $45 \mathrm{~m}$ telescope, we observed 13 Planck cold clumps, for which we have already obtained accurate positions from preliminary IRAM $30 \mathrm{~m}$ observations in $\mathrm{N}_{2} \mathrm{H}^{+}$and/or SCUBA-2 observations. We selected sources whose $\mathrm{C}^{18} \mathrm{O} J=1-0$ linewidths are relatively narrow $(<1.5$ $\mathrm{km} \mathrm{s}^{-1}$ ) from the previous observations with the $13.7 \mathrm{~m}$ telescope of the Purple Mountain Observatory (PMO) at De

\footnotetext{
${ }^{25}$ http://radio.kasi.re.kr/trao/key_science.php
}

Ling Ha, because we prefer to observe relatively close objects to investigate the chemical differentiation on scales of 0.05-0.1 pc (Ohashi et al. 2014, 2016a; Tatematsu et al. 2014b). However, some of our sources (G108.8-00.8 and $\mathrm{G} 120.7+2.7)$ are actually distant $(1-3.5 \mathrm{kpc})$, which needs some care in discussion based on the column density ratios. While we focus on the chemical evolution stage as the main target of the current study, we also briefly investigate the physical properties of the sources by analyzing the specific angular momenta.

\section{Observations}

\subsection{James Clerk Maxwell Telescope}

Observations with the $15 \mathrm{~m}$ JCMT on Mauna Kea were made between 2014 November and 2015 December in the pilot survey phase (project IDs: M15AI05, M15BI061) of the JCMT legacy survey program "SCOPE." The Submillimetre Common-User Bolometer Array 2 (SCUBA-2) was employed for observations of the $850 \mu \mathrm{m}$ continuum. It is a 10,000 pixel bolometer camera operating simultaneously at 450 and 850 $\mu \mathrm{m}$. Observations were carried out in constant velocity (CV) Daisy mode under grade $3 / 4$ weather conditions with a $225 \mathrm{GHz}$ opacity between 0.1 and 0.15 . The mapping area is about $12^{\prime} \times 12^{\prime}$. The beam size of SCUBA-2 at $850 \mu \mathrm{m}$ is $\sim 14^{\prime \prime}$. The typical rms noise level of the maps is about 6-10 mJy beam ${ }^{-1}$ in the central $3^{\prime}$ area, and increases to $10-30 \mathrm{mJy}^{-}$beam $^{-1}$ out to $12^{\prime}$. The data were reduced using SMURF in the STARLINK package.

\subsection{Nobeyama $45 \mathrm{~m}$ Telescope}

Observations with the $45 \mathrm{~m}$ radio telescope of Nobeyama Radio Observatory ${ }^{26}$ were carried out from 2015 December to 2016 February. We observed eight molecular lines by using the receivers TZ1 and T70 (Table 1). Observations with the receiver TZ1 (we used one beam called TZ1 out of two beams of the receiver TZ) (Asayama \& Nakajima 2013; Nakajima et al. 2013) were made to simultaneously observe four molecular lines, $82 \mathrm{GHz} \mathrm{CCS}, 94 \mathrm{GHz} \mathrm{CCS}, \mathrm{HC}_{3} \mathrm{~N}$, and $\mathrm{N}_{2} \mathrm{H}^{+}$. Observations were carried out with $\mathrm{T} 70$ to simultaneously observe four other lines, $\mathrm{HN}^{13} \mathrm{C}, \mathrm{DNC}, \mathrm{N}_{2} \mathrm{D}^{+}$, and c- $\mathrm{C}_{3} \mathrm{H}_{2}$. TZ1 and T70 are double-polarization, two-sideband SIS receivers. Molecular transitions are selected to achieve a high angular resolution of $\sim 20^{\prime \prime}$ to differentiate the distribution of molecules, and they are detectable even from cold gas $(\lesssim 20 \mathrm{~K})$. The upper energy level $E_{u}$ of the observed transitions are listed in Table 1.

The full width at half maximum (FWHM) beam sizes at $86 \mathrm{GHz}$ with TZ1 and T70 were 18". $2 \pm 0$ ". 1 and 18 ". $8 \pm 0$ ". 3 , respectively. The main-beam efficiency $\eta_{m b}$ at $86 \mathrm{GHz}$ with TZ1 was $54 \pm 3 \%$ and $53 \pm 3 \%$ in $\mathrm{H}$ and $\mathrm{V}$ polarizations, respectively. The efficiencies with $\mathrm{T} 70$ was $54 \pm 3 \%$ and $55 \pm 3 \%$ in $\mathrm{H}$ and $\mathrm{V}$ polarizations, respectively. We also observed $\mathrm{NH}_{3}(J, K)=(1,1)$ at $23.694495 \mathrm{GHz}$ (Ho \& Townes 1983) in both circular polarizations with the receiver $\mathrm{H} 22$. The FWHM beam size at $23 \mathrm{GHz}$ with $\mathrm{H} 22$ was 74 ". $4 \pm$ 0 ." 3 and 73 ". $9 \pm 0$ ". 3 for ch1 and ch2 (right-handed and lefthanded circular polarization), respectively. The main-beam efficiency $\eta_{m b}$ at $23 \mathrm{GHz}$ with $\mathrm{H} 22$ was $83 \pm 4 \%$ and

\footnotetext{
26 Nobeyama Radio Observatory is a branch of the National Astronomical Observatory of Japan, National Institutes of Natural Sciences.
} 
Table 1

Observed Lines

\begin{tabular}{|c|c|c|c|c|c|}
\hline Line & Frequency & Frequency Reference & Upper Energy Level $E_{u}$ & Receiver & Observing Mode \\
\hline $\operatorname{CCS} J_{N}=7_{6}-6_{5}$ & $81.505208 \mathrm{GHz}$ & Cummins et al. (1986) & $15.3 \mathrm{~K}$ & $\mathrm{TZ1}$ & OTF \\
\hline $\operatorname{CCS} J_{N}=8_{7}-6_{5}$ & $93.870107 \mathrm{GHz}$ & Yamamoto et al. (1990) & $19.9 \mathrm{~K}$ & $\mathrm{TZ1}$ & OTF \\
\hline $\mathrm{HC}_{3} \mathrm{~N} J=9-8$ & $81.8814614 \mathrm{GHz}$ & Picket et al. (1998) & $19.7 \mathrm{~K}$ & $\mathrm{TZ1}$ & OTF \\
\hline $\mathrm{N}_{2} \mathrm{H}^{+} J=1-0$ & $93.1737767 \mathrm{GHz}$ & Caselli \& Myers (1995) & $4.5 \mathrm{~K}$ & $\mathrm{TZ1}$ & OTF \\
\hline DNC $J=1-0$ & $76.3057270 \mathrm{GHz}$ & Picket et al. (1998) & $3.7 \mathrm{~K}$ & T70 & single pointing \\
\hline $\mathrm{HN}^{13} \mathrm{C} J=1-0$ & $87.090859 \mathrm{GHz}$ & Frerking et al. (1979) & $4.2 \mathrm{~K}$ & $\mathrm{~T} 70$ & single pointing \\
\hline $\mathrm{N}_{2} \mathrm{D}^{+} J=1-0$ & $77.1096100 \mathrm{GHz}$ & Picket et al. (1998) & $3.7 \mathrm{~K}$ & $\mathrm{~T} 70$ & single pointing \\
\hline c- $\mathrm{C}_{3} \mathrm{H}_{2} J_{K_{a} K_{c}}=2_{12}-1_{01}$ & $85.338906 \mathrm{GHz}$ & Thaddeus et al. (1981) & $4.1 \mathrm{~K}$ & $\mathrm{~T} 70$ & single pointing \\
\hline $\mathrm{NH}_{3}(J, K)=(1,1)$ & $23.694495 \mathrm{GHz}$ & Ho \& Townes (1983) & $23.4 \mathrm{~K}$ & $\mathrm{H} 22$ & single pointing \\
\hline
\end{tabular}

$84 \pm 4 \%$, for $\mathrm{ch} 1$ and $\mathrm{ch} 2$, respectively. The receiver backend was the digital spectrometer "SAM45." The spectral resolution was $15.26 \mathrm{kHz}$ (corresponding to $0.05-0.06 \mathrm{~km} \mathrm{~s}^{-1}$ ) for TZ1 and $\mathrm{T} 70$, and $3.81 \mathrm{kHz}$ (corresponding to $\sim 0.05 \mathrm{~km} \mathrm{~s}^{-1}$ ) for $\mathrm{H} 22$.

Observations with receiver TZ1 were conducted in the onthe-fly (OTF) mapping mode (Sawada et al. 2008) with data sampling intervals along a strip of $5^{\prime \prime}$ and separations between strips of $5^{\prime \prime}$. We have made orthogonal scans in R.A. and Decl. to minimize scan effects. Observations with receivers T70 and $\mathrm{H} 22$ were carried out in the ON-OFF position-switching mode. The ON positions with receiver T70 were determined from possible intensity peaks in $\mathrm{N}_{2} \mathrm{H}^{+}$on temporal lower-S/N-ratio TZ1 maps during observations (before completion), but are not necessarily intensity peaks in $\mathrm{N}_{2} \mathrm{H}^{+}$on the final maps. In addition, the $\mathrm{T} 70$ position of $\mathrm{G} 207 \mathrm{~N}$ was incorrect.

Coordinates used for the observations are listed in Table 2. The distances to the sources are taken from Ramirez Alegria et al. (2011) for G108.8-00.8, Wu et al. (2012) for G120.7 +2.7 , Lombardi et al. (2010) for G157.6-12.2, Loinard et al. (2007) and Lombardi et al. (2010) for G174.0-15.8, Kim et al. (2008) for G192.33-11.88, G202.31-8.92, G204.4-11.3, and G207.3-19.8, and Clariá (1974) for G224.4-0.6. The distances to G089.9-01.9, G149.5-1.2, and G202.00+2.65 are taken from Planck Collaboration XXVIII (2016).

The observed intensity is reported in terms of the corrected antenna temperature $T_{A}^{*}$. To derive the physical parameters, we use the main-beam radiation temperature $T_{m b}=T_{A}^{*} / \eta_{m b}$. The telescope pointing was established by observing relevant $43-\mathrm{GHz} \mathrm{SiO}$ maser sources every $\sim 60$ minutes for TZ1 and T70 observations, and was accurate to $\sim 5^{\prime \prime}$. For H22 observations, pointing was established once a day in the beginning of observations. The observed data were reduced using the software package "NoStar" and "NewStar" of Nobeyama Radio Observatory.

\section{Results}

Figures 1 through 13 show the maps obtained with JCMT/ SCUBA-2 and with the Nobeyama $45 \mathrm{~m}$ telescope. Table 3 lists the dense cores detected with JCMT/SCUBA-2, their intensities, and column densities. The column density is derived by using Equation (A.27) in Kauffmann (2007). Associations of young stars are investigated on the basis of WISE $22 \mu \mathrm{m}$, Akari, Spitzer images, and IRAS point source catalog, and they are classified through their spectral indexes (Liu et al. 2016, in preparation). $\mathrm{N}_{2} \mathrm{H}^{+}$was detected for all sources, and we detected $81 \mathrm{GHz}$ CCS from 7 out of 13 . Tables 4 and 5 summarize observed parameters from
Nobeyama $45 \mathrm{~m}$ telescope receiver TZ1. The emission line spectra have a single velocity component, when they are detected, and there is no position showing two velocity components. For Table 5, we should note that T70 positions do not necessarily coincide with the final $\mathrm{N}_{2} \mathrm{H}^{+}$peaks as intended. The line parameters are obtained through Gaussian fitting (either single-component Gaussian or hyperfine fitting). The upper limit to the intensity is defined as $3 \sigma$, where $\sigma$ is the rms noise level at $1 \mathrm{~km} \mathrm{~s}^{-1}$ bin.

Tables 6 summarizes the observed parameters from the Nobeyama $45 \mathrm{~m}$ telescope receiver T70. Again, emission line spectra have single velocity component, when they are detected, and there is no position showing two velocity components. We detected DNC, $\mathrm{HN}^{13} \mathrm{C}, \mathrm{c}-\mathrm{C}_{3} \mathrm{H}_{2}$ all but G108N and G207N (again, G207N position was mistakenly set). The $\mathrm{N}_{2} \mathrm{D}^{+}$hyperfine transitions were detected toward four out of eight sources (excluding G207N).

Furthermore, we observed 15 sources with the Nobeyama $45 \mathrm{~m}$ telescope receiver $\mathrm{H} 22$ in $\mathrm{NH}_{3}$ transitions in singlepointing observations. Nine out of them were detected in $(J, K)=(2,2)$, and we derived $T_{\text {rot }}$. The $\mathrm{NH}_{3}$ rotation temperature $T_{\text {rot }}$ is derived as explained in Ho \& Townes (1983), and then converted to the gas kinetic temperature $T_{k}$ by using the relation by Tafalla et al. (2004). Observed intensities, derived $T_{\text {rot }}$ and $T_{k}$ are summarized in Table 7 .

We investigate the molecular line intensity distribution shown in Figures 1 to 13. In general, the $\mathrm{N}_{2} \mathrm{H}^{+}$distribution (black contours in panel (b)) is quite similar to the $850 \mu \mathrm{m}$ dust continuum emission distribution (contours in panel (a); gray-scale in panels (b) and (c)). The $82 \mathrm{GHz}$ CCS emission (blue contours) is clumpy in general, and is often located at the edge of the $\mathrm{N}_{2} \mathrm{H}^{+}$/ $850 \mu \mathrm{m}$ core or is distributed as it surrounds the $\mathrm{N}_{2} \mathrm{H}^{+} / 850 \mu \mathrm{m}$ core. We draw the $2.5 \sigma$ contour in some cases to let the reader understand the reliability of the $3 \sigma$ contour as detection. Most clumps are as cold as 10-20 K, and therefore the depletion of CCS can contribute to a configuration of the $\mathrm{N}_{2} \mathrm{H}^{+}$core being surrounded by CCS (Aikawa et al. 2001; Bergin et al. 2002). The $94 \mathrm{GHz}$ CCS emission, when detected, does not necessarily follow that of the $82 \mathrm{GHz}$ CCS emission, although the upper energy levels of these transitions do not differ so much. Taking the beamsizes of JCMT/SCUBA-2 and the Nobeyama $45 \mathrm{~m}$ telescope and the pointing accuracy of the latter telescope, only differences in the spatial distribution larger than $10^{\prime \prime}$ will be meaningful.

The molecular column density is calculated by assuming local thermodynamic equilibrium (LTE) as explained in, e.g., Suzuki et al. (1992), Mangum \& Shirley (2015), Sanhueza et al. (2012). $\mathrm{N}_{2} \mathrm{H}^{+}, \mathrm{N}_{2} \mathrm{D}^{+}$, and $\mathrm{NH}_{3}$ have hyperfine transitions, and we can derive the excitation temperatures $T_{\text {ex }}$ directly from 
Table 2

Coordinates for the Observations

\begin{tabular}{|c|c|c|c|c|c|c|c|c|c|c|}
\hline Source & $\begin{array}{l}\text { OTF Reference Center } \\
\text { R.A.(J2000.0) }\end{array}$ & Decl.(J2000.0) & OTF area & $\begin{array}{c}\text { T70 } \\
\text { R.A.(J2000.0) }\end{array}$ & Decl.(J2000.0) & $\begin{array}{c}\mathrm{H} 22 \\
\text { R.A.(J2000.0) }\end{array}$ & Decl.(J2000.0) & $\begin{array}{c}\text { OFF } \\
\text { R.A.(J2000.0) }\end{array}$ & Decl.(J2000.0) & $\begin{array}{l}\text { Distance } \\
(\mathrm{kpc})\end{array}$ \\
\hline G089.9-01.9 & $21: 20: 04.35$ & $46: 54: 46.8$ & $2^{\prime} \times 2^{\prime}$ & $21: 20: 04.35$ & $46: 54: 46.8$ & $21: 20: 04.35$ & $46: 54: 46.8$ & $21: 19: 20$ & $46: 54: 00$ & 0.62 \\
\hline G108.8-00.8N (G108N) & $22: 58: 56.2$ & $58: 58: 21.7$ & $2^{\prime} \times 2^{\prime}$ & $22: 58: 53.6$ & $58: 58: 21.7$ & $22: 58: 56.2$ & $58: 58: 21.7$ & $22: 58: 15$ & 59:01:00 & 3.2 \\
\hline G108.8-00.8S (G108S) & $22: 58: 40.8$ & $58: 55: 34.4$ & $2^{\prime} \times 2^{\prime}$ & $\ldots$ & $\ldots$ & $22: 58: 40.8$ & $58: 55: 34.4$ & $22: 58: 15$ & 59:01:00 & 3.2 \\
\hline $\mathrm{G} 120.7+2.7 \mathrm{~N}(\mathrm{G} 120 \mathrm{~N})$ & $00: 29: 26.11$ & $65: 27: 14.3$ & $2^{\prime} \times 2^{\prime}$ & $00: 29: 29.3$ & $65: 27: 14.3$ & $00: 29: 26.11$ & $65: 27: 14.3$ & $00: 29: 20$ & $65: 20: 00$ & 1.82 \\
\hline G120.7+2.7S (G120S) & $00: 29: 46.76$ & $65: 25: 47.8$ & $2^{\prime} \times 2^{\prime}$ & $\ldots$ & $\ldots$ & $00: 29: 46.76$ & $65: 25: 47.8$ & $00: 29: 20$ & $65: 20: 00$ & 1.82 \\
\hline G149.5-1.2 & $03: 56: 53$ & $51: 48: 00$ & $3^{\prime} \times 3^{\prime}$ & $03: 56: 57.3$ & 51:49:00 & $03: 56: 57.3$ & $51: 49: 00$ & $03: 56: 15$ & $51: 44: 00$ & 0.84 \\
\hline G157.6-12.2 & $03: 51: 53.6$ & $38: 15: 22.6$ & $2^{\prime} \times 2^{\prime}$ & 03:51:53.6 & $38: 15: 22.6$ & $03: 51: 53.6$ & $38: 15: 22.6$ & $03: 51: 20$ & $38: 10: 00$ & 0.45 \\
\hline G174.0-15.8 & $04: 32: 45.30$ & $24: 21: 51.7$ & $2^{\prime} \times 2^{\prime}$ & $\ldots$ & $\ldots$ & $04: 32: 45.30$ & $24: 21: 51.7$ & 04:33:09.19 & $24: 23: 42.7$ & 0.15 \\
\hline G192.33-11.88 & 05:29:54.16 & $12: 16: 53.0$ & $2^{\prime} \times 2^{\prime}$ & 05:29:54.16 & $12: 16: 53.0$ & 05:29:54.16 & $12: 16: 53.0$ & 05:30:05 & $12: 10: 00$ & 0.42 \\
\hline G202.00+2.65 & $\ldots$ & $\ldots$ & $\ldots$ & $\ldots$ & $\ldots$ & $06: 40: 55.10$ & $10: 56: 16.2$ & $06: 40: 43.93$ & $11: 01: 40.8$ & 0.76 \\
\hline G202.31-8.92 & $\ldots$ & $\ldots$ & $\ldots$ & $\ldots$ & $\ldots$ & 06:00:10.0 & 05:15:00.0 & $06: 00: 18$ & 05:20:00 & 0.42 \\
\hline G204.4-11.3 & $05: 55: 38.54$ & 02:11:35.6 & $2^{\prime} \times 2^{\prime}$ & $05: 55: 38.54$ & 02:11:35.6 & $05: 55: 38.54$ & 02:11:35.6 & $05: 55: 48.98$ & 02:05:37.0 & 0.42 \\
\hline $\mathrm{G} 207.3-19.8 \mathrm{~N}(\mathrm{G} 207 \mathrm{~N})$ & 05:30:48 & $-04: 11: 30$ & $4^{\prime} \times 4^{\prime}$ & $05: 30: 48.5$ & $-04: 09: 40.0$ & $05: 30: 46.5$ & $-04: 10: 30$ & $05: 31: 35$ & $-04: 12: 22$ & 0.42 \\
\hline G207.3-19.8S (G207S) & 05:31:03.00 & $-04: 16: 20$ & $4^{\prime} \times 4^{\prime}$ & $\ldots$ & $\ldots$ & 05:31:03.00 & $-04: 17.05 .3$ & $05: 31: 35$ & $-04: 12: 22$ & 0.42 \\
\hline G224.4-0.6 & $07: 10: 03.83$ & $-10: 31: 28.21$ & $2^{\prime} \times 2^{\prime}$ & 07:09:59.8 & $-10: 31: 18.2$ & 07:10:01.12 & $-10: 30: 58.2$ & 07:09:55 & $-10: 28: 00$ & 1.1 \\
\hline
\end{tabular}

Note. Three dots means "not observed." 

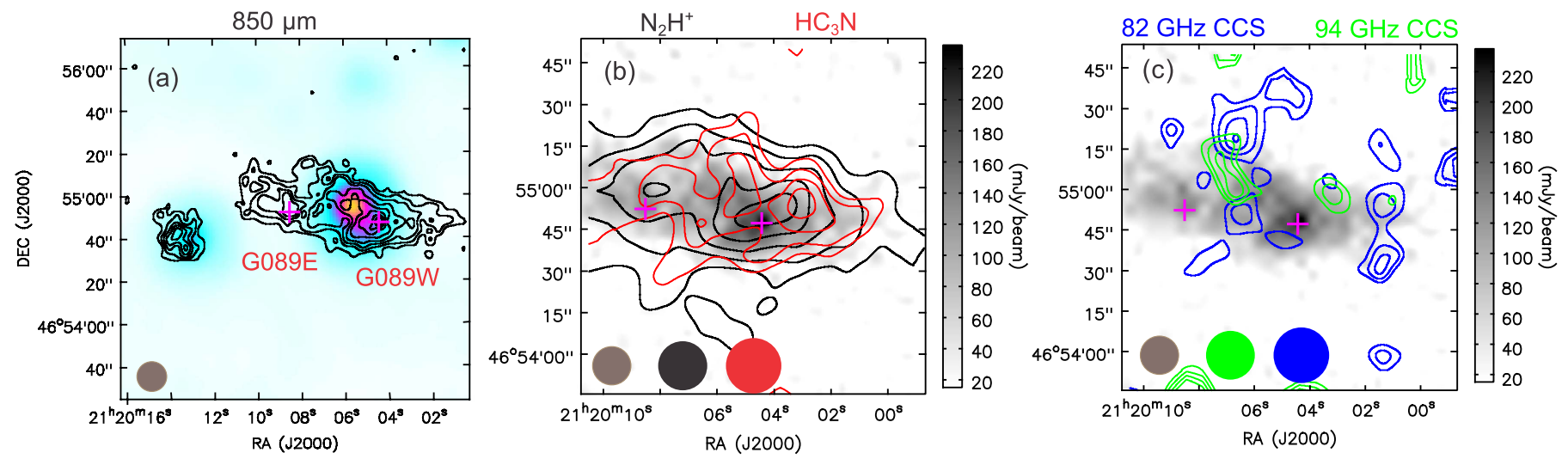

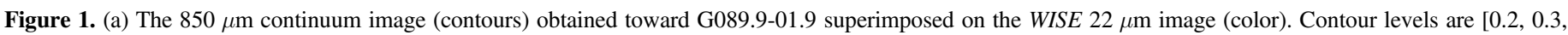

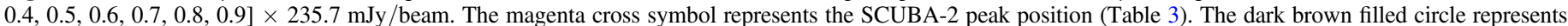

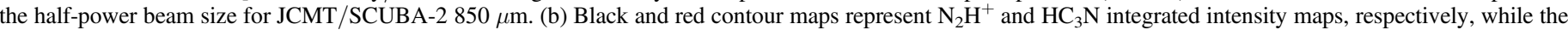

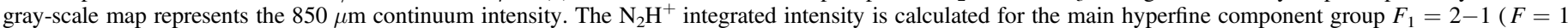

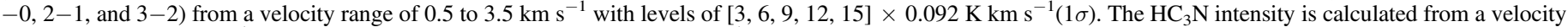

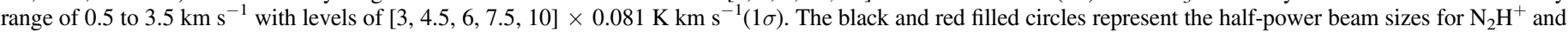

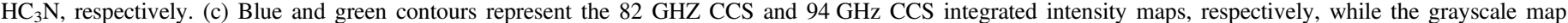

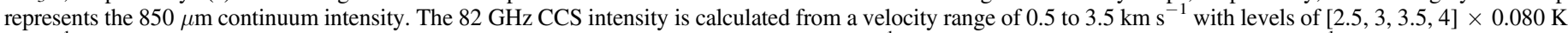

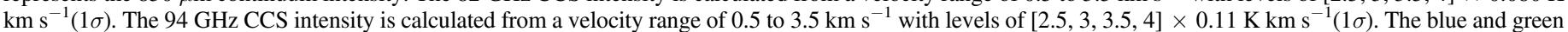
filled circles represent the half-power beam sizes for $82 \mathrm{GHz}$ CCS and $94 \mathrm{GHz} \mathrm{CCS}$, respectively.

observations. We assume that the beam filling factor is unity. If the filling factor is lower than unity, we underestimate $T_{\mathrm{ex}} \cdot T_{\mathrm{ex}}$ from $\mathrm{N}_{2} \mathrm{H}^{+}$ranges from 3.7 to $5.7 \mathrm{~K}$. When we compared $T_{\mathrm{ex}}$ for $\mathrm{N}_{2} \mathrm{H}^{+}$with $T_{K}$ derived from $\mathrm{NH}_{3}$ observations, we obtain a relation of $T_{\mathrm{ex}}=0.45 \pm 0.27 T_{K}$. We assume that the excitation temperatures $T_{\text {ex }}$ for the other molecular lines are $0.5 T_{K}$, assuming that the levels are subthermally excited to $50 \%$ (for $\mathrm{G} 207 \mathrm{~N}$, we assume $T_{\mathrm{ex}}=0.6 T_{K}$ so that the observed intensity can be explained in LTE). If $T_{k}$ is an upper limit, we assume $T_{k}=10 \mathrm{~K}$. The necessary parameters for CCS are taken from Yamamoto et al. (1990) and references therein. For $\mathrm{N}_{2} \mathrm{H}^{+}$, if the hyperfine fitting is unsuccessful, we try to calculate the column density by using the velocity-integrated intensity of a main $F_{1}=2-1$ group of the three hyperfine components ( $F=1-0,2-1$, and $3-2$ ), by assuming optically thin emission, and by neglecting the background term.

We show in Table 8 the column density range by assuming that the actual column density is a factor of 1-10 larger than the optically thin estimate. The resulting column densities are listed in Tables 8 and 9. Although we observed the $94 \mathrm{GHz}$ CCS emission, the detection rate is not high. At T70 positions, only two clumps were detected but the $\mathrm{S} / \mathrm{N}$ ratio is low. We decided to use $T_{k}$ to estimate $T_{\mathrm{ex}}$ (CCS) instead of the excitation analysis (e.g., Large Velocity Gradient models) to treat all the data in a consistent way. Tables 10 and 11 list the fractional abundance $X$ of molecules relative to $\mathrm{H}_{2}$ calculated from the column density ratio toward the JCMT/SCUBA-2 peaks and T70 positions, respectively. The $\mathrm{H}_{2}$ column density is taken from the dust continuum flux density measured toward the T70 position.

In the next section, we introduce the results for individual sources.

\section{Individual Objects}

\subsection{G089.9-01.9}

The object is located in L974 (Lynds 1962; Dobashi et al. 2005), and in the dark cloud Khavtassi 137 (Khavtassi 1960). There is a Class 0 like source (IRAS 21182+4611), which is a bright source in the WISE image, northeast of the western core G089W with an offset of $\sim 15^{\prime \prime}$. Then, we regard G089W itself as starless. To our knowledge, there is no information that the eastern core G089E is associated with any young stellar object, and we also regard it as starless. Figure 1 shows maps toward G089.9-01.9. The $\mathrm{N}_{2} \mathrm{H}^{+}$distribution shows a resemblance to the $850 \mu \mathrm{m}$ distribution, but their peak positions are slightly different. The $\mathrm{HC}_{3} \mathrm{~N}$ distribution shows some similarity to the $850 \mu \mathrm{m}$ distribution, but the correlation between their intensities is poor. The $82 \mathrm{GHz}$ CCS distribution is very clumpy, and appears as if it surrounds the $850 \mu \mathrm{m}$ core. The $94 \mathrm{GHz}$ CCS distribution is different, but it is still located on both sides of the $850 \mu \mathrm{m}$ core. Nobeyama T70 observations were made toward G089W. The column density ratio of $N(\mathrm{DNC})$ to $N\left(\mathrm{HN}^{13} \mathrm{C}\right)$ is 4.5 , which is close to the value of 3.0 at L1544 (Hirota et al. 2003; Hirota \& Yamamoto 2006). L1544 is known as a prestellar core showing collapsing motion (Tafalla et al. 1998).

\section{2. $6108.8-00.8$}

G108.8-00.8 is located in a GMC $\left(1^{\circ} \times 15^{\prime}\right)$ associated with five Sharpless (1959) HII regions, S147, S148, S149, S152, and S153, and also associated with the supernova remnant G109.11.0 (CTB109) in the Perseus arm (Tatematsu et al. 1985, 1987). These two cores are located between peaks $\alpha$ (corresponding to S152) and $\zeta$ in Tatematsu et al. (1985). G108.8-00.8S is associated with two Class I-like sources seen in the WISE image, while G108.8-00.8N is starless. Figure 2 shows maps toward G108.8-00.8. The $\mathrm{N}_{2} \mathrm{H}^{+}$distribution resembles the $850 \mu \mathrm{m}$ distribution. The $\mathrm{HC}_{3} \mathrm{~N}$ emission is observed at the edge of the $850 \mu \mathrm{m}$ core of $\mathrm{G} 108 \mathrm{~N}$, and the correlation between $\mathrm{N}_{2} \mathrm{H}^{+}$and $\mathrm{HC}_{3} \mathrm{~N}$ is poor. For G108S, their distributions are more or less correlated. The $82 \mathrm{GHz}$ CCS emission is detected toward the $\mathrm{N}_{2} \mathrm{H}^{+} / 850 \mu \mathrm{m}$ cores $(\mathrm{G} 108 \mathrm{~N}$ and G108S) and also the edge of them $(\mathrm{G} 108 \mathrm{~N})$. Note that this source is distant, the spatial resolution is as large as $0.3 \mathrm{pc}$. It is possible that different distributions between $\mathrm{N}_{2} \mathrm{H}^{+}$and CCS are less clear due to its large distance. The kinetic temperature of 
Table 3

Parameters of SCUBA-2 Peaks

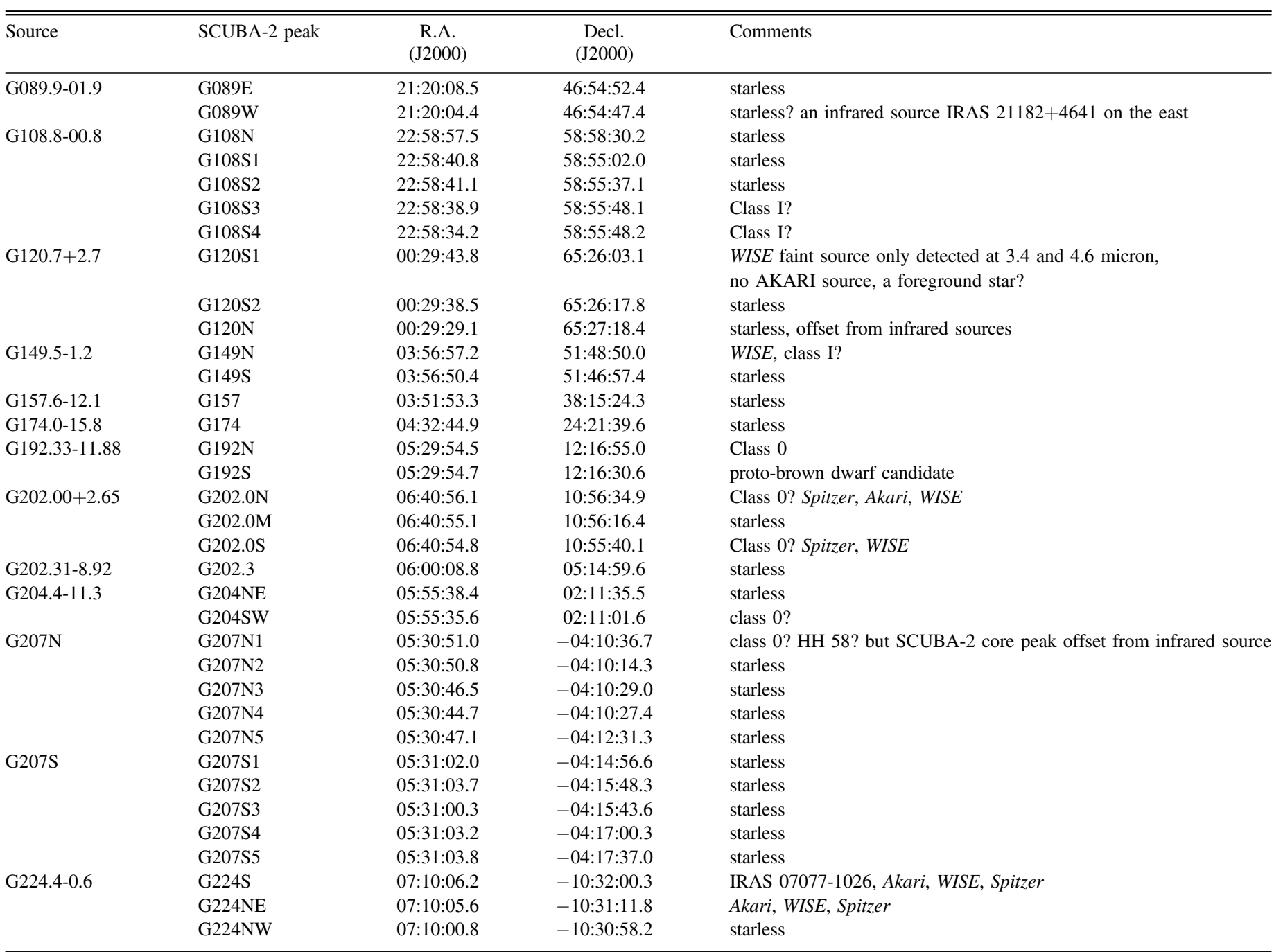

G108S is $14.3 \pm 3.0 \mathrm{~K}$. G108S is as cold as typical cold dark clouds. Nobeyama T70 observations were made toward G108N, a starless core. We detected DNC, but not $\mathrm{HN}^{13} \mathrm{C}$. The column density ratio of $N(\mathrm{DNC})$ to $N\left(\mathrm{HN}^{13} \mathrm{C}\right)$ is $>2.5$, which is similar to the value of 3.0 at L1544 (Hirota et al. 2003; Hirota \& Yamamoto 2006). Kim et al. (2016) will report our observations with other telescopes such as PMO $14 \mathrm{~m}, \mathrm{CSO}$, IRAM $30 \mathrm{~m}$ telescopes, etc.

\section{3. $G 120.7+2.7$}

Figure 3 shows maps toward G120.7+2.7. The WISE source is clearly offset from G120N, and we regard the latter as a starless core. Both $\mathrm{N}_{2} \mathrm{H}^{+}$and $\mathrm{HC}_{3} \mathrm{~N}$ distributions are well correlated with the $850 \mu \mathrm{m}$ distribution. In G120N, the $82 \mathrm{GHz}$ CCS emission has two intensity peaks, and one of them is close to the $\mathrm{N}_{2} \mathrm{H}^{+}$emission peak. Toward the $\mathrm{N}_{2} \mathrm{H}^{+}$peak, we detected $\mathrm{N}_{2} \mathrm{D}^{+}$. In G120S, the $\mathrm{N}_{2} \mathrm{H}^{+}$emission shows two cores. We clearly detected both $82 \mathrm{GHz}$ and $94 \mathrm{GHz} \mathrm{CCS}$ emission at G120S. It is possible that the CCS emission surrounds the $\mathrm{N}_{2} \mathrm{H}^{+}$cores, although it is less clear. Nobeyama T70 observations were made toward G120N, a starless core.
The column density ratio of $N(\mathrm{DNC})$ to $N\left(\mathrm{HN}^{13} \mathrm{C}\right)$ is 1.7 , which is close to the value of 1.91 at L1498 (Hirota \& Yamamoto 2006).

\subsection{G149.5-1.2}

This clump is located at the edge of the dark cloud Khavtassi 241 (Khavtassi 1960). Figure 4 shows maps toward G149.51.2. The $\mathrm{N}_{2} \mathrm{H}^{+}$distribution is well correlated with the $850 \mu \mathrm{m}$ distribution. The $\mathrm{HC}_{3} \mathrm{~N}$ emission is poorly correlated with the $850 \mu \mathrm{m}$ distribution, and it is located on both sides of the northeastern $850 \mu \mathrm{m}$ (and $\mathrm{N}_{2} \mathrm{H}^{+}$) core. We detected the $\mathrm{N}_{2} \mathrm{D}^{+}$ emission in single pointing observations between the brightest $\mathrm{N}_{2} \mathrm{H}^{+}$core G149N (WISE source) and the northern $\mathrm{HC}_{3} \mathrm{~N}$ core. The column density ratio of $N(\mathrm{DNC})$ to $N\left(\mathrm{HN}^{13} \mathrm{C}\right)$ is 3.2 , which is close to the value of 3.0 in L1544 (Hirota et al. 2003; Hirota \& Yamamoto 2006).

\subsection{G157.6-12.2}

This clump is located near the dark cloud L1449 (Lynds 1962; Dobashi et al. 2005) and in Khavtassi 257 (Khavtassi 1960), which is close to the California Nebula, NGC 
Table 4

Intensities ${ }^{\mathrm{a}}$ Observed with the Receiver TZ1 toward the SCUBA-2 Peaks

\begin{tabular}{|c|c|c|c|c|c|c|c|c|c|c|c|c|c|c|c|}
\hline $\begin{array}{l}\text { SCUBA- } \\
2 \text { peak }\end{array}$ & $\begin{array}{c}82 \\
\mathrm{GHz} \mathrm{CCS} \\
T_{A}^{*} \\
(\mathrm{~K})\end{array}$ & $\begin{array}{c}V_{\mathrm{LSR}} \\
\left(\mathrm{km} \mathrm{s}^{-1}\right)\end{array}$ & $\begin{array}{c}\Delta v \\
\left(\mathrm{~km} \mathrm{~s}^{-1}\right)\end{array}$ & $\begin{array}{c}94 \\
\mathrm{GHz} \mathrm{CCS} \\
T_{A}^{*} \\
(\mathrm{~K})\end{array}$ & $\begin{array}{c}V_{\mathrm{LSR}} \\
\left(\mathrm{km} \mathrm{s}^{-1}\right)\end{array}$ & $\begin{array}{c}\Delta v \\
\left(\mathrm{~km} \mathrm{~s}^{-1}\right)\end{array}$ & $\begin{array}{c}\mathrm{HC}_{3} \mathrm{~N} \\
T_{A}^{*} \\
(\mathrm{~K})\end{array}$ & $\begin{array}{c}V_{\mathrm{LSR}} \\
\left(\mathrm{km} \mathrm{s}^{-1}\right)\end{array}$ & $\begin{array}{c}\Delta v \\
\left(\mathrm{~km} \mathrm{~s}^{-1}\right)\end{array}$ & $\begin{array}{c}\mathrm{N}_{2} \mathrm{H}^{+} \\
T_{A}^{*} \\
(\mathrm{~K})\end{array}$ & $\begin{array}{c}V_{\mathrm{LSR}} \\
\left(\mathrm{km} \mathrm{s}^{-1}\right)\end{array}$ & $\begin{array}{c}\Delta v \\
\left(\mathrm{~km} \mathrm{~s}^{-1}\right)\end{array}$ & $T_{\mathrm{ex}}$ & $\tau($ main $)$ & $\begin{array}{l}\text { Integrated } \\
\text { Intensity } \\
\left(\mathrm{K} \mathrm{km} \mathrm{s}^{-1}\right)\end{array}$ \\
\hline$\overline{\text { G089E }}$ & 0.60 & 1.94 & 0.14 & 0.27 & 1.80 & 0.68 & 0.56 & 1.82 & 0.49 & 0.8 & 1.82 & 0.40 & $4.6 \pm 0.4$ & $6.2 \pm 2.0$ & $\ldots$ \\
\hline G089W & 0.51 & 1.31 & 0.33 & 0.43 & 1.43 & 0.42 & 1.08 & 1.29 & 0.47 & 1.1 & 1.50 & 0.63 & $8.7 \pm 4.2$ & $1.4 \pm 1.2$ & $\ldots$ \\
\hline G108N & 0.18 & -49.38 & 1.53 & $<0.16$ & $\ldots$ & $\ldots$ & $\ldots$ & $\ldots$ & $\ldots$ & 0.5 & -49.40 & 1.20 & $5.8 \pm 3.8$ & $0.9 \pm 1.3$ & $\ldots$ \\
\hline G108S1 & 0.19 & -49.00 & 0.78 & $\ldots$ & $\ldots$ & $\ldots$ & 0.28 & -48.84 & 0.33 & $<0.10$ & $\ldots$ & $\ldots$ & $\ldots$ & $\ldots$ & $\ldots$ \\
\hline G108S2 & 0.18 & -49.58 & 1.44 & $\ldots$ & $\ldots$ & $\ldots$ & 0.22 & -49.66 & 1.39 & 0.3 & -49.78 & 1.30 & $3.4 \pm 0.2$ & $3.2 \pm 1.5$ & $\ldots$ \\
\hline G108S3 & $<0.12$ & $\ldots$ & $\ldots$ & $\ldots$ & $\ldots$ & $\ldots$ & $\ldots$ & $\ldots$ & $\ldots$ & 0.2 & $\sim-50$ & $\ldots$ & $\ldots$ & $\ldots$ & 0.5 \\
\hline G108S4 & $<0.12$ & $\ldots$ & $\ldots$ & $\ldots$ & $\ldots$ & $\ldots$ & $\ldots$ & $\ldots$ & $\ldots$ & 0.3 & -51.20 & 1.22 & $3.8 \pm 0.8$ & $1.9 \pm 1.9$ & $\ldots$ \\
\hline G120S1 & 0.25 & -18.01 & 1.48 & 0.21 & -18.12 & 1.48 & 0.51 & -18.22 & 1.27 & 0.6 & -18.33 & 1.33 & $4.8 \pm 0.6$ & $1.9 \pm 0.7$ & $\ldots$ \\
\hline G120S2 & $<0.14$ & $\ldots$ & $\cdots$ & $<0.15$ & $\cdots$ & $\cdots$ & 0.79 & -18.16 & 0.92 & 0.4 & -18.06 & 0.98 & $3.9 \pm 0.3$ & $4.0 \pm 2.0$ & $\cdots$ \\
\hline G120N & 0.21 & -18.44 & 0.95 & $<0.17$ & $\cdots$ & $\cdots$ & 0.39 & -18.57 & 1.20 & 1.2 & -18.46 & 1.24 & $22.7 \pm 29.0$ & $0.3 \pm 0.5$ & 2.6 \\
\hline G149N & $<0.16$ & $\ldots$ & $\cdots$ & $<0.19$ & $\cdots$ & $\cdots$ & $\cdots$ & $\ldots$ & $\cdots$ & 0.6 & -7.51 & 0.41 & $4.5 \pm 1.3$ & $3.5 \pm 3.3$ & $\cdots$ \\
\hline G149S & $<0.16$ & $\ldots$ & $\ldots$ & $<0.19$ & $\ldots$ & $\ldots$ & $\cdots$ & $\cdots$ & $\cdots$ & $<0.17$ & $\ldots$ & $\ldots$ & $\cdots$ & $\cdots$ & $\cdots$ \\
\hline G157 & 0.57 & -7.62 & 0.28 & $<0.19$ & $\ldots$ & $\cdots$ & $\cdots$ & $\cdots$ & $\cdots$ & 0.8 & -7.63 & 0.29 & $4.2 \pm 0.1$ & $16.5 \pm 5.0$ & $\cdots$ \\
\hline G174 & 0.55 & 6.27 & 0.33 & $<0.16$ & $\cdots$ & $\cdots$ & $\cdots$ & $\cdots$ & $\cdots$ & 0.7 & $6-7$ & $\cdots$ & $\ldots$ & $\cdots$ & 0.5 \\
\hline G192N & $<0.16$ & $\cdots$ & $\cdots$ & $<0.17$ & $\cdots$ & $\cdots$ & $\cdots$ & $\cdots$ & $\cdots$ & 0.6 & $\sim 6$ & $\cdots$ & $\cdots$ & $\cdots$ & 1.4 \\
\hline G192S & $<0.16$ & $\cdots$ & $\cdots$ & $<0.17$ & $\cdots$ & $\cdots$ & 0.37 & 12.17 & 0.75 & 1.3 & 12.23 & 0.55 & $5.7 \pm 0.2$ & $6.2 \pm 0.9$ & $\cdots$ \\
\hline G204NE & 0.38 & 1.74 & 0.71 & $<0.17$ & $\ldots$ & $\ldots$ & $\ldots$ & $\cdots$ & $\cdots$ & 1.6 & 1.57 & 0.47 & $6.0 \pm 0.4$ & $7.5 \pm 1.6$ & $\cdots$ \\
\hline G204SW & $<0.21$ & $\ldots$ & $\ldots$ & $<0.20$ & $\ldots$ & $\ldots$ & $\ldots$ & $\cdots$ & $\cdots$ & 0.5 & 1.74 & 0.76 & $5.2 \pm 3.1$ & $1.8 \pm 2.9$ & 1.0 \\
\hline G207N1 & $<0.22$ & $\cdots$ & $\ldots$ & $<0.22$ & $\ldots$ & $\cdots$ & 0.44 & 10.48 & 1.20 & 0.9 & 10.71 & 1.10 & $6.1 \pm 1.8$ & $2.4 \pm 1.8$ & $\cdots$ \\
\hline G207N2 & $<0.22$ & $\cdots$ & $\cdots$ & $<0.22$ & $\cdots$ & $\cdots$ & $\cdots$ & $\cdots$ & $\ldots$ & 0.8 & 11.20 & 0.45 & $5.6 \pm 1.6$ & $3.6 \pm 2.8$ & $\cdots$ \\
\hline G207N3 & $<0.22$ & $\ldots$ & $\ldots$ & $<0.22$ & $\ldots$ & $\ldots$ & $\ldots$ & $\cdots$ & $\cdots$ & & $\ldots$ & $\ldots$ & $\ldots$ & $\ldots$ & $\ldots$ \\
\hline G207N4 & $<0.22$ & $\ldots$ & $\ldots$ & $<0.22$ & $\ldots$ & $\ldots$ & $\ldots$ & $\ldots$ & $\ldots$ & 0.4 & 11.28 & 0.54 & $3.5 \pm 0.1$ & $21.5 \pm 12.4$ & 0.9 \\
\hline G207N5 & $<0.22$ & $\ldots$ & $\ldots$ & $<0.22$ & $\ldots$ & $\ldots$ & $\ldots$ & $\ldots$ & $\ldots$ & 0.9 & $\sim 11$ & $\ldots$ & $\ldots$ & $\ldots$ & 0.7 \\
\hline G224S & 0.22 & 14.44 & 2.59 & $<0.19$ & $\ldots$ & $\ldots$ & 0.49 & 14.4 & 2.17 & 1.5 & $\sim 15$ & $\ldots$ & $\ldots$ & $\ldots$ & 3.9 \\
\hline G224NE & 0.15 & 13.87 & 3.46 & $<0.19$ & $\ldots$ & $\ldots$ & 0.36 & 13.75 & 1.46 & 1.6 & 13.95 & 1.46 & $13.6 \pm 4.2$ & $0.9 \pm 0.4$ & $\ldots$ \\
\hline G224NW & 0.23 & 16.21 & 3.04 & $<0.19$ & $\ldots$ & $\ldots$ & 0.74 & 16.38 & 2.00 & 0.6 & $\sim 16$ & $\ldots$ & $\ldots$ & $\ldots$ & 0.2 \\
\hline
\end{tabular}

Notes.

${ }^{\text {a }}$ The upper limit to the intensity is defined as $3 \sigma$, where $\sigma$ is the rms noise level at $1 \mathrm{~km} \mathrm{~s}^{-1}$ bin.

Three dots in $T_{A}^{*}$ means "hard to judge whether the emission is detected or not, due to low signal-to-noise ratios, baseline distortion, and/or possible spurious emission". 
Table 5

Intensities ${ }^{\mathrm{a}}$ Observed with the Receiver TZ1 toward T70 Position

\begin{tabular}{|c|c|c|c|c|c|c|c|c|c|c|c|c|c|c|}
\hline \multirow[t]{2}{*}{ Source } & \multirow{2}{*}{$\begin{array}{c}82 \mathrm{GHz} \mathrm{CCS} \\
T_{A}^{*} \\
(\mathrm{~K})\end{array}$} & \multirow[b]{2}{*}{$\begin{array}{c}V_{\mathrm{LSR}} \\
\left(\mathrm{km} \mathrm{s}^{-1}\right)\end{array}$} & \multicolumn{3}{|c|}{$94 \mathrm{GHz} \mathrm{CCS}$} & \multirow[b]{2}{*}{$\begin{array}{c}\Delta v \\
\left(\mathrm{~km} \mathrm{~s}^{-1}\right)\end{array}$} & \multirow{2}{*}{$\begin{array}{c}\mathrm{HC}_{3} \mathrm{~N} \\
T_{A}^{*} \\
(\mathrm{~K})\end{array}$} & \multirow[b]{2}{*}{$\begin{array}{c}V_{\mathrm{LSR}} \\
\left(\mathrm{km} \mathrm{s}^{-1}\right)\end{array}$} & \multirow[b]{2}{*}{$\begin{array}{c}\Delta v \\
\left(\mathrm{~km} \mathrm{~s}^{-1}\right)\end{array}$} & \multicolumn{3}{|l|}{$\mathrm{N}_{2} \mathrm{H}^{+}$} & \multirow[b]{2}{*}{$\begin{array}{l}T_{\mathrm{ex}} \\
(\mathrm{K})\end{array}$} & \multirow[b]{2}{*}{$\tau($ main $)$} \\
\hline & & & $\begin{array}{c}\Delta v \\
\left(\mathrm{~km} \mathrm{~s}^{-1}\right)\end{array}$ & $\begin{array}{l}T_{A}^{*} \\
(\mathrm{~K})\end{array}$ & $\begin{array}{c}V_{\mathrm{LSR}} \\
\left(\mathrm{km} \mathrm{s}^{-1}\right)\end{array}$ & & & & & $\begin{array}{l}T_{A}^{*} \\
(\mathrm{~K})\end{array}$ & $\begin{array}{c}V_{\mathrm{LSR}} \\
\left(\mathrm{km} \mathrm{s}^{-1}\right)\end{array}$ & $\begin{array}{c}\Delta v \\
\left(\mathrm{~km} \mathrm{~s}^{-1}\right)\end{array}$ & & \\
\hline G089.9-01.9 & 0.53 & 1.32 & 0.32 & 0.44 & 1.44 & 0.47 & 1.23 & 1.29 & 0.45 & 1.1 & 1.49 & 0.60 & $9.0 \pm 4.9$ & $1.4 \pm 1.3$ \\
\hline G108N & 0.16 & -49.62 & 1.56 & $<0.16$ & $\cdots$ & $\cdots$ & $<0.12$ & $\cdots$ & $\cdots$ & 0.5 & -49.89 & 1.39 & $\cdots$ & $\cdots$ \\
\hline G120N & 0.21 & -18.44 & 0.95 & $<0.17$ & $\cdots$ & $\cdots$ & 0.45 & -18.65 & 0.81 & 1.2 & -18.57 & 1.13 & $8.1 \pm 1.5$ & $1.6 \pm 0.6$ \\
\hline G120S & 0.35 & -18.42 & 0.73 & 0.33 & -18.42 & 0.31 & 0.80 & -18.47 & 0.66 & $<0.14$ & $\cdots$ & $\cdots$ & $\cdots$ & $\cdots$ \\
\hline G149.5-1.2 & $<0.16$ & $\cdots$ & $\cdots$ & $<0.19$ & $\cdots$ & $\cdots$ & $<0.14$ & $\cdots$ & $\cdots$ & 0.6 & -7.51 & 0.41 & $4.5 \pm 1.3$ & $3.5 \pm 3.3$ \\
\hline G157.6-12.2 & 0.59 & -7.62 & 0.27 & $<0.20$ & $\cdots$ & $\cdots$ & $<0.15$ & $\cdots$ & $\cdots$ & 0.9 & -7.61 & 0.29 & $4.5 \pm 0.2$ & $9.4 \pm 1.9$ \\
\hline G174.0-15.8 & 0.64 & 6.27 & 0.44 & $<0.16$ & $\cdots$ & $\cdots$ & $<0.11$ & $\cdots$ & $\cdots$ & $<0.15$ & $\cdots$ & $\cdots$ & $\cdots$ & $\cdots$ \\
\hline G192.33-11.88 & $<0.16$ & $\cdots$ & $\cdots$ & $<0.17$ & 13.48 & 0.37 & $<0.15$ & $\cdots$ & $\cdots$ & 0.7 & 12.12 & 1.13 & $4.9 \pm 0.3$ & $2.7 \pm 0.6$ \\
\hline G204.4-11.3 & 0.38 & 1.74 & 0.71 & $<0.20$ & $\cdots$ & $\cdots$ & $<0.18$ & $\cdots$ & $\cdots$ & 1.4 & 1.59 & 0.47 & $5.6 \pm 0.2$ & $8.6 \pm 1.5$ \\
\hline G207N $N^{b}$ & $<0.22$ & $\cdots$ & $\cdots$ & $<0.24$ & $\cdots$ & $\cdots$ & $<0.19$ & $\cdots$ & $\cdots$ & $<0.23$ & $\cdots$ & $\ldots$ & $\cdots$ & $\ldots$ \\
\hline G224.4-0.6 & $<0.18$ & $\cdots$ & $\cdots$ & $<0.19$ & $\cdots$ & $\cdots$ & $<0.17$ & $\cdots$ & $\cdots$ & 1.6 & 13.95 & 1.46 & $13.6 \pm 4.2$ & $0.9 \pm 0.4$ \\
\hline
\end{tabular}

Notes.

${ }^{\text {a }}$ The upper limit to the intensity is defined as $3 \sigma$, where $\sigma$ is the rms noise level at $1 \mathrm{~km} \mathrm{~s}^{-1}$ bin.

${ }^{\mathrm{b}}$ Incorrect position. 
Table 6

Intensities ${ }^{\mathrm{a}}$ Observed with the Receiver T70

\begin{tabular}{|c|c|c|c|c|c|c|c|c|c|c|c|c|c|c|}
\hline \multirow[t]{2}{*}{ Source } & \multirow{2}{*}{$\begin{array}{c}\mathrm{DNC} \\
T_{A}^{*} \\
(\mathrm{~K})\end{array}$} & \multirow[b]{2}{*}{$\begin{array}{c}V_{\mathrm{LSR}} \\
\left(\mathrm{km} \mathrm{s}^{-1}\right)\end{array}$} & \multirow[b]{2}{*}{$\underset{\left(\mathrm{km} \mathrm{s}^{-1}\right)}{\Delta v}$} & \multicolumn{3}{|l|}{$\mathrm{HN}^{13} \mathrm{C}$} & \multicolumn{3}{|l|}{$\overline{\mathrm{N}} \mathrm{N}_{2} \mathrm{D}^{+}$} & \multirow[b]{2}{*}{$\begin{array}{l}T_{\mathrm{ex}} \\
(\mathrm{K})\end{array}$} & \multirow[b]{2}{*}{$\tau(\mathrm{tau})$} & \multicolumn{2}{|c|}{ ce- $\mathrm{C}_{3} \mathrm{H}_{2}$} & \multirow[b]{2}{*}{$\underset{\left(\mathrm{km} \mathrm{s}^{-1}\right)}{\Delta v}$} \\
\hline & & & & $\begin{array}{l}T_{A}^{*} \\
(\mathrm{~K})\end{array}$ & $\begin{array}{c}V_{\mathrm{LSR}} \\
\left(\mathrm{km} \mathrm{s}^{-1}\right)\end{array}$ & $\underset{\left(\mathrm{km} \mathrm{s}^{-1}\right)}{\Delta v}$ & $\begin{array}{l}T_{A}^{*} \\
(\mathrm{~K})\end{array}$ & $\begin{array}{c}V_{\mathrm{LSR}} \\
\left(\mathrm{km} \mathrm{s}^{-1}\right)\end{array}$ & $\underset{\left(\mathrm{km} \mathrm{s}^{-1}\right)}{\Delta v}$ & & & $\begin{array}{l}T_{A}^{*} \\
(\mathrm{~K})\end{array}$ & $\begin{array}{c}V_{\mathrm{LSR}} \\
\left(\mathrm{km} \mathrm{s}^{-1}\right)\end{array}$ & \\
\hline G089.9-01.9 & 0.90 & 1.49 & 1.19 & 0.44 & 1.49 & 0.79 & 0.23 & 1.50 & 0.50 & $3.7 \pm 0.4$ & $2.09 \pm 1.08$ & 0.69 & 1.39 & 0.78 \\
\hline G108N & 0.18 & -49.46 & 2.48 & $<0.089$ & $\ldots$ & 2.48 & $<0.036$ & $\ldots$ & $\ldots$ & $\ldots$ & $\ldots$ & 0.21 & -49.84 & 0.95 \\
\hline G120N & 0.30 & -18.52 & 1.51 & 0.25 & -18.46 & 1.353 & $0.05<0.036$ & $\cdots$ & $\cdots$ & $\cdots$ & $\cdots$ & 0.51 & -18.45 & 1.41 \\
\hline G149.5-1.2 & 0.17 & -7.47 & 1.00 & 0.12 & -7.31 & 0.592 & $0.05<0.036$ & $\cdots$ & $\cdots$ & $\cdots$ & $\ldots$ & 0.28 & -7.44 & 0.46 \\
\hline G157.6-12.2 & 1.34 & -7.66 & 0.90 & 0.53 & -7.55 & 0.564 & 0.45 & -7.65 & 0.31 & $4.1 \pm 0.2$ & $3.42 \pm 0.66$ & 1.25 & -7.61 & 0.35 \\
\hline G192.33-11.88 & 0.81 & 12.14 & 1.06 & 0.22 & 12.32 & 0.959 & 0.28 & 12.16 & 0.59 & $5.8 \pm 2.0$ & $0.58 \pm 0.40$ & 0.42 & 12.19 & 0.84 \\
\hline G204.4-11.3 & 1.69 & 1.64 & 1.03 & 0.74 & 1.70 & 0.79 & 0.38 & 1.63 & 0.52 & $4.7 \pm 1.4$ & $1.68 \pm 1.33$ & 1.47 & 1.64 & 0.66 \\
\hline G207N $N^{b}$ & $<0.040$ & $\ldots$ & $\ldots$ & $<0.026$ & $\ldots$ & & $<0.036$ & $\ldots$ & $\ldots$ & $\ldots$ & $\ldots$ & 0.05 & 11.12 & 1.64 \\
\hline G224.4-0.6 & 0.23 & 15.76 & 1.42 & 0.17 & 15.73 & 0.479 & $<0.044$ & $\cdots$ & $\cdots$ & $\ldots$ & $\ldots$ & 0.19 & 15.69 & 1.35 \\
\hline
\end{tabular}

\section{Notes.}

${ }^{\mathrm{a}}$ The upper limit to the intensity is defined as $3 \sigma$, where $\sigma$ is the rms noise level at $1 \mathrm{~km} \mathrm{~s}^{-1}$ bin. 
Table 7

$\mathrm{NH}_{3}$ Intensities ${ }^{\mathrm{a}}$ Observed with the Receiver $\mathrm{H} 22$ and Derived Rotation and Kinetic Temperatures

\begin{tabular}{|c|c|c|c|c|c|c|}
\hline Source & $\begin{array}{c}\mathrm{NH}_{3}(1,1) \\
T_{A}^{*} \\
(\mathrm{~K})\end{array}$ & $\begin{array}{c}V_{\mathrm{LSR}} \\
\left(\mathrm{km} \mathrm{s}^{-1}\right)\end{array}$ & $\begin{array}{c}\Delta v \\
\left(\mathrm{~km} \mathrm{~s}^{-1}\right)\end{array}$ & $\begin{array}{c}\mathrm{NH}_{3}(2,2) \\
T_{A}^{*} \\
(\mathrm{~K})\end{array}$ & $\begin{array}{l}T_{\text {rot }} \\
(\mathrm{K})\end{array}$ & $\begin{array}{l}T_{k} \\
(\mathrm{~K})\end{array}$ \\
\hline G089.9-01.9 & 0.87 & 1.59 & 0.86 & 0.11 & $10.5 \pm 0.9$ & $11.0 \pm 1.1$ \\
\hline G108S & 0.22 & -49.82 & 2.06 & $<0.03$ & $<16.5$ & $<19.1$ \\
\hline G120N & 0.42 & -18.39 & 1.61 & 0.11 & $13.1 \pm 4.1$ & $14.3 \pm 5.4$ \\
\hline G120S & 0.57 & -18.47 & 1.11 & 0.15 & $11.3 \pm 1.5$ & $12.1 \pm 1.9$ \\
\hline G174.0-15.8 & 0.99 & 6.19 & 0.81 & $<0.03$ & $<+10.5$ & $<11.1$ \\
\hline G192.33-11.88 & 1.01 & 12.06 & 0.90 & 0.21 & $11.6 \pm 1.7$ & $12.5 \pm 2.1$ \\
\hline $\mathrm{G} 202.00+2.65$ & 0.80 & 5.08 & 0.77 & $<0.06$ & $<9.8$ & $<10.2$ \\
\hline G202.31-8.92 & 0.60 & 11.92 & 0.89 & $<0.03$ & $<10.9$ & $<11.6$ \\
\hline G204.4-11.3 & 1.34 & 1.56 & 0.73 & 0.14 & $9.8 \pm 0.7$ & $10.2 \pm 0.8$ \\
\hline G207N & 0.87 & 11.04 & 1.04 & 0.14 & $12.9 \pm 5.6$ & $14.1 \pm 7.3$ \\
\hline
\end{tabular}

Note.

${ }^{\text {a }}$ The upper limit to the intensity is defined as $3 \sigma$, where $\sigma$ is the rms noise level at $1 \mathrm{~km} \mathrm{~s}^{-1}$ bin.

Table 8

Column Densities and Their Ratios toward SCUBA-2 Peak

\begin{tabular}{|c|c|c|c|}
\hline SCUBA-2 peak & $\begin{array}{l}N(\mathrm{CCS}) \\
\left(\mathrm{cm}^{-2}\right)\end{array}$ & $\begin{array}{c}N\left(\mathrm{~N}_{2} \mathrm{H}^{+}\right) \\
\left(\mathrm{cm}^{-2}\right)\end{array}$ & $N\left(\mathrm{~N}_{2} \mathrm{H}^{+}\right) / N(\mathrm{CCS})$ \\
\hline G089E & $3.5 \mathrm{E}+12$ & $1.2 \mathrm{E}+13$ & 3.6 \\
\hline G089W & $6.4 \mathrm{E}+12$ & $4.5 \mathrm{E}+12$ & 0.7 \\
\hline G108N & $5.2 \mathrm{E}+12$ & $5.4 \mathrm{E}+12$ & 1.0 \\
\hline G108S1 & $6.4 \mathrm{E}+12$ & & \\
\hline G108S2 & $1.1 \mathrm{E}+13$ & $2.5 \mathrm{E}+13$ & 2.3 \\
\hline G108S3 & $<4.9 \mathrm{E}+12$ & $1.7 \mathrm{E}+12-1.7 \mathrm{E}+13$ & $>0.3$ \\
\hline G108S4 & $<4.9 \mathrm{E}+12$ & $1.3 \mathrm{E}+13$ & $>2.6$ \\
\hline G120S1 & $9.8 \mathrm{E}+12$ & $1.3 \mathrm{E}+13$ & 1.3 \\
\hline G120S2 & $<3.5 \mathrm{E}+12$ & $2.1 \mathrm{E}+13$ & $>5.9$ \\
\hline G120N & $3.8 \mathrm{E}+12$ & $3.5 \mathrm{E}+12$ & 0.9 \\
\hline G149N & $<6.8 \mathrm{E}+12$ & $7.2 \mathrm{E}+12$ & $>1.1$ \\
\hline G149S & $<6.8 \mathrm{E}+12$ & & $\ldots$ \\
\hline G157 & $8.9 \mathrm{E}+12$ & $2.5 \mathrm{E}+13$ & 2.8 \\
\hline G174 & $5.0 \mathrm{E}+12$ & $1.7 \mathrm{E}+12-1.7 \mathrm{E}+13$ & $0.3-3.3$ \\
\hline G192N & $<3.9 \mathrm{E}+12$ & $4.6 \mathrm{E}+12-4.6 \mathrm{E}+13$ & $>1.2$ \\
\hline G192S & $<6.9 \mathrm{E}+12$ & $1.7 \mathrm{E}+13$ & $>2.4$ \\
\hline G204NE & $1.2 \mathrm{E}+13$ & $1.7 \mathrm{E}+13$ & 1.4 \\
\hline G204SW & $\ldots$ & $6.7 \mathrm{E}+12$ & \\
\hline G207N1 & $<3.3 \mathrm{E}+12$ & $1.2 \mathrm{E}+13$ & $>3.8$ \\
\hline G207N2 & $<3.3 \mathrm{E}+12$ & $7.7 \mathrm{E}+12$ & $>2.3$ \\
\hline G207N3 & $<3.3 \mathrm{E}+12$ & & $\cdots$ \\
\hline G207N4 & $<3.3 \mathrm{E}+12$ & $6.8 \mathrm{E}+13$ & $>21$ \\
\hline G207N5 & $<3.3 \mathrm{E}+12$ & $2.6 \mathrm{E}+12-2.6 \mathrm{E}+13$ & $>0.8$ \\
\hline $\mathrm{G} 224 \mathrm{~S}$ & $9.6 \mathrm{E}+12$ & $1.4 \mathrm{E}+13-1.4 \mathrm{E}+14$ & $1.5-15$ \\
\hline G224NE & $8.6 \mathrm{E}+12$ & $7.7 \mathrm{E}+12$ & 0.9 \\
\hline G224NW & $1.2 \mathrm{E}+13$ & $6.4 \mathrm{E}+11-6.4 \mathrm{E}+12$ & $0.05-0.5$ \\
\hline
\end{tabular}

1499. Figure 5 shows maps toward G157.6-12.2. The $\mathrm{N}_{2} \mathrm{H}^{+}$ distribution is well correlated with the $850 \mu \mathrm{m}$ distribution. The $82 \mathrm{GHz}$ CCS emission surrounds the $850 \mu \mathrm{m}$ (and $\mathrm{N}_{2} \mathrm{H}^{+}$) core. The $\mathrm{HC}_{3} \mathrm{~N}$ emission shows distribution different from the $\mathrm{N}_{2} \mathrm{H}^{+}$distribution, and looks anticorrelated with the $82 \mathrm{GHz}$ CCS emission. Nobeyama T70 observations were made toward the center of G157, a starless core. The column density ratio of $N(\mathrm{DNC})$ to $N\left(\mathrm{HN}^{13} \mathrm{C}\right)$ is 6.9 , which is larger than the value of 3.0 at L1544 (Hirota et al. 2003; Hirota \& Yamamoto 2006).

\section{6. $G 174.0-15.8$}

This clump is located in L1529 (Lynds 1962; Wouterloot \& Habing 1985; Dobashi et al. 2005) in Taurus. G174.0-15.8 is a starless clump. Figure 6 shows maps toward G174.0-15.8. The $\mathrm{N}_{2} \mathrm{H}^{+}$distribution is more or less correlated with the $850 \mu \mathrm{m}$ distribution. The $\mathrm{HC}_{3} \mathrm{~N}$ and $82 \mathrm{GHz}$ CCS emission is distributed more extensively than the $850 \mu \mathrm{m}$ core.

\section{7. $G 192.32-11.88$}

G192.3-11.8 is located in the $\lambda$ Orionis complex, and is associated with B30 cataloged by Barnard (1927), L1581 (Lynds 1962; Dobashi et al. 2005), and with No. 9 CO emission peak identified by Maddalena et al. (1986). It is located in the dark cloud Khavtassi 296 (Khavtassi 1960). Liu et al. (2016) discovered an extremely young Class 0 protostellar object (G192N) and a proto-brown dwarf candidate (G192S). Observations with SMA show the existence of molecular outflows associated with these objects. Figure 7 shows maps toward G192.32-11.88. The $\mathrm{N}_{2} \mathrm{H}^{+}$distribution shows an intensity peak toward G192S, while there is no $\mathrm{N}_{2} \mathrm{H}^{+}$peak corresponding to G192N. The $850 \mu \mathrm{m}$ map of SCUBA-2 has two clear peaks, G192N being the more intense one. The $\mathrm{HC}_{3} \mathrm{~N}$ distribution is very different from that of $850 \mu \mathrm{m}$ (and $\mathrm{N}_{2} \mathrm{H}^{+}$), and is clumpy. G192S is associated with one $\mathrm{HC}_{3} \mathrm{~N}$ core, while there is no $\mathrm{HC}_{3} \mathrm{~N}$ counterpart to $\mathrm{G} 192 \mathrm{~N}$. It seems that the $850 \mu \mathrm{m}$ (and $\mathrm{N}_{2} \mathrm{H}^{+}$) peak is surrounded by $\mathrm{HC}_{3} \mathrm{~N}$ cores.

Liu et al. (2016) detected large velocity gradient in this region in ${ }^{13} \mathrm{CO} J=1-0$ and $2-1$ in the NE-SW direction, and attributed its origin to compression by the $\mathrm{H}$ II region. The $\mathrm{N}_{2} \mathrm{H}^{+}$core inside shows an E-W velocity gradient of the order of $0.5 \mathrm{~km} \mathrm{~s}^{-1} \operatorname{arcmin}^{-1}$ or $4 \mathrm{~km} \mathrm{~s}^{-1} \mathrm{pc}^{-1}$. This gradient is consistent with what was observed by Liu et al. (2016) toward the center of their Clump-S in ${ }^{13} \mathrm{CO} J=1-0$ and 2-1 (see their Figure 2). Figure 14 shows the intensity-weighted radial velocity (moment 1) map toward G192.32-11.88 in the $\mathrm{N}_{2} \mathrm{H}^{+}$ main hyperfine emission group $F_{1}=2-1(F=1-0,2-1$, and 3-2). This shows a velocity gradient of $0.5 \mathrm{~km} \mathrm{~s}^{-1}$ across the clump in the $\mathrm{E}-\mathrm{W}$ direction. Because this object is located 
Table 9

Column Densities and Their Ratios toward T70 or H22 Positions

\begin{tabular}{|c|c|c|c|c|c|c|c|c|c|c|}
\hline Source & $\begin{array}{l}N(\mathrm{CCS}) \\
\left(\mathrm{cm}^{-2}\right)\end{array}$ & $\begin{array}{c}N\left(\mathrm{~N}_{2} \mathrm{H}^{+}\right) \\
\left(\mathrm{cm}^{-2}\right)\end{array}$ & $\begin{array}{c}N\left(\mathrm{~N}_{2} \mathrm{D}^{+}\right) \\
\left(\mathrm{cm}^{-2}\right)\end{array}$ & $\begin{array}{c}N\left(\mathrm{HN}^{13} \mathrm{C}\right) \\
\left(\mathrm{cm}^{-2}\right)\end{array}$ & $\begin{array}{c}N(\mathrm{DNC}) \\
\left(\mathrm{cm}^{-2}\right)\end{array}$ & $\begin{array}{l}N\left(\mathrm{NH}_{3}\right) \\
\left(\mathrm{cm}^{-2}\right)\end{array}$ & $N\left(\mathrm{~N}_{2} \mathrm{H}^{+}\right) / N(\mathrm{CCS})$ & $N(\mathrm{DNC}) / N\left(\mathrm{HN}^{13} \mathrm{C}\right)$ & $N\left(\mathrm{~N}_{2} \mathrm{D}^{+}\right) / N\left(\mathrm{~N}_{2} \mathrm{H}^{+}\right)$ & $N\left(\mathrm{NH}_{3}\right) / N(\mathrm{CCS})$ \\
\hline G089.9-01.9 & $6.5 \mathrm{E}+12$ & $4.2 \mathrm{E}+12$ & $5.9 \mathrm{E}+12$ & $1.5 \mathrm{E}+12$ & $7.6 \mathrm{E}+12$ & $5.2 \mathrm{E}+14 \pm 9.5 \mathrm{E}+13$ & 0.7 & 5.0 & 1.4 & $80 \pm 15$ \\
\hline G108N & $4.8 \mathrm{E}+12$ & & $\cdots$ & $<7.5 \mathrm{E}+11$ & $1.9 \mathrm{E}+12$ & $5.1 \mathrm{E}+14 \pm 1.7 \mathrm{E}+14$ & $\cdots$ & $>2.5$ & $\cdots$ & $106 \pm 36$ \\
\hline G108S & $\cdots$ & & $\cdots$ & & $\cdots$ & $<2.6 \mathrm{E}+14$ & $\cdots$ & $\cdots$ & $\cdots$ & $\cdots$ \\
\hline G120N & $3.8 \mathrm{E}+12$ & $8.9 \mathrm{E}+12$ & $\cdots$ & $1.2 \mathrm{E}+12$ & $2.0 \mathrm{E}+12$ & $4.0 \mathrm{E}+14 \pm 2.0 \mathrm{E}+14$ & 2.4 & 1.7 & $\cdots$ & $106 \pm 53$ \\
\hline G120S & $7.0 \mathrm{E}+12$ & & $\ldots$ & & $\cdots$ & $5.2 \mathrm{E}+14 \pm 1.4 \mathrm{E}+14$ & $\cdots$ & $\cdots$ & $\cdots$ & $74 \pm 19$ \\
\hline G149.5-1.2 & $<3.0 \mathrm{E}+12$ & $7.2 \mathrm{E}+12$ & $\ldots$ & $2.3 \mathrm{E}+11$ & $7.4 \mathrm{E}+11$ & $<1.9 \mathrm{E}+14$ & $>2.4$ & 3.2 & $\cdots$ & $\cdots$ \\
\hline G157.6-12.2 & $5.7 \mathrm{E}+12$ & $1.4 \mathrm{E}+13$ & $5.7 \mathrm{E}+12$ & $1.3 \mathrm{E}+12$ & $1.4 \mathrm{E}+13$ & $<2.4 \mathrm{E}+14$ & 2.4 & 10.4 & 0.4 & $<42$ \\
\hline G174.0-15.8 & $1.1 \mathrm{E}+13$ & $<7.0 \mathrm{E}+12$ & $\cdots$ & & $\cdots$ & $<1.9 \mathrm{E}+14$ & $<0.6$ & $\cdots$ & $\cdots$ & $<17$ \\
\hline G192.33-11.88 & $<4.8 \mathrm{E}+12$ & $1.5 \mathrm{E}+13$ & $1.6 \mathrm{E}+12$ & $7.8 \mathrm{E}+11$ & $4.8 \mathrm{E}+12$ & $3.4 \mathrm{E}+14 \pm 9.2 \mathrm{E}+13$ & $>3.1$ & 6.2 & 0.1 & $>71$ \\
\hline G202.00+2.65 & $\cdots$ & & $\cdots$ & & $\cdots$ & $<5.1 \mathrm{E}+14$ & $\cdots$ & $\cdots$ & $\cdots$ & $\cdots$ \\
\hline G202.31-8.92 & $\ldots$ & & $\ldots$ & & $\ldots$ & $<2.6 \mathrm{E}+14$ & $\ldots$ & $\ldots$ & $\ldots$ & $\ldots$ \\
\hline G204.4-11.3 & $1.2 \mathrm{E}+13$ & $2.0 \mathrm{E}+13$ & $4.3 \mathrm{E}+12$ & $3.5 \mathrm{E}+12$ & $\cdots$ & $4.6 \mathrm{E}+14 \pm 7.8 \mathrm{E}+13$ & 1.6 & $\cdots$ & 0.2 & $37 \pm 6$ \\
\hline $\mathrm{G} 207 \mathrm{~N}^{\mathrm{a}}$ & $<6.3 \mathrm{E}+12$ & & $\cdots$ & & $\cdots$ & $2.1 \mathrm{E}+14 \pm 1.5 \mathrm{E}+14$ & $\cdots$ & $\cdots$ & $\cdots$ & $\cdots$ \\
\hline G207S & $\cdots$ & & $\cdots$ & & $\cdots$ & $2.3 \mathrm{E}+14 \pm 2.1 \mathrm{E}+14$ & $\cdots$ & $\cdots$ & $\cdots$ & $\cdots$ \\
\hline G224.4-0.6 & $<2.7 \mathrm{E}+12$ & $7.7 \mathrm{E}+12$ & $\cdots$ & & $\cdots$ & $\cdots$ & $>2.9$ & $\cdots$ & $\cdots$ & $\cdots$ \\
\hline
\end{tabular}

\section{Note.}

${ }^{\mathrm{a}}$ Incorrect position. 
Table 10

Fractional Abundance toward the JCMT/SCUBA-2 Peak

\begin{tabular}{|c|c|c|c|c|}
\hline Source & $\begin{array}{c}S_{\nu}^{\text {beam a }} \\
(\mathrm{mJy} / \text { beam })\end{array}$ & $\begin{array}{c}N_{\mathrm{H}_{2}} \\
\left(10^{22} \mathrm{~cm}^{-2}\right)\end{array}$ & $X(\mathrm{CCS})$ & $X\left(\mathrm{~N}_{2} \mathrm{H}^{+}\right)$ \\
\hline G089E & 132.2 & 1.7 & $2.0 \mathrm{E}-10$ & $7.3 \mathrm{E}-10$ \\
\hline G089W & 235.7 & 3.0 & $2.1 \mathrm{E}-10$ & $1.5 \mathrm{E}-10$ \\
\hline G108N & 231.9 & 1.8 & $2.9 \mathrm{E}-10$ & $3.0 \mathrm{E}-10$ \\
\hline G108S1 & 167.3 & 0.8 & $8.0 \mathrm{E}-10$ & $\ldots$ \\
\hline G108S2 & 197.5 & 1.0 & $1.1 \mathrm{E}-09$ & $2.5 \mathrm{E}-09$ \\
\hline G108S3 & 175.9 & 0.9 & $\ldots$ & $\ldots$ \\
\hline G108S4 & 141.7 & 0.7 & $\ldots$ & $1.8 \mathrm{E}-09$ \\
\hline G120S1 & 332.1 & 3.5 & $2.8 \mathrm{E}-10$ & $3.6 \mathrm{E}-10$ \\
\hline G120S2 & 116.9 & 1.2 & $\ldots$ & $1.7 \mathrm{E}-09$ \\
\hline G120N & 514.4 & 4.1 & $9.3 \mathrm{E}-11$ & $8.7 \mathrm{E}-11$ \\
\hline G149N & 117.2 & 0.9 & $\ldots$ & $8.0 \mathrm{E}-10$ \\
\hline G149S & 93.2 & 0.7 & $\ldots$ & $\ldots$ \\
\hline G157 & 126.8 & 1.5 & $5.9 \mathrm{E}-10$ & $1.7 \mathrm{E}-09$ \\
\hline G174 & 90.3 & 1.1 & $4.5 \mathrm{E}-10$ & $\ldots$ \\
\hline G192N & 600.3 & 6.0 & $\ldots$ & $\ldots$ \\
\hline G192S & 333.3 & 3.3 & $\ldots$ & $5.0 \mathrm{E}-10$ \\
\hline G204NE & 861.5 & 12.8 & $9.5 \mathrm{E}-11$ & $1.3 \mathrm{E}-10$ \\
\hline G204SW & 338.5 & 5.0 & $\ldots$ & $1.3 \mathrm{E}-10$ \\
\hline G207N1 & 386.4 & 3.1 & $\ldots$ & $4.0 \mathrm{E}-10$ \\
\hline G207N2 & 216.2 & 1.7 & $\ldots$ & $4.5 \mathrm{E}-10$ \\
\hline G207N3 & 185.3 & 1.5 & $\ldots$ & $\ldots$ \\
\hline G207N4 & 163.7 & 1.3 & $\cdots$ & $5.2 \mathrm{E}-09$ \\
\hline G207N5 & 121.9 & 1.0 & $\ldots$ & $\ldots$ \\
\hline G224S & 940.3 & 6.5 & $1.5 \mathrm{E}-10$ & $\ldots$ \\
\hline G224NE & 946.5 & 6.5 & $1.3 \mathrm{E}-10$ & $1.2 \mathrm{E}-10$ \\
\hline G224NW & 387.5 & 2.7 & $4.4 \mathrm{E}-10$ & $\cdots$ \\
\hline Median & 206.9 & 1.7 & $2.8 \mathrm{E}-10$ & $4.5 \mathrm{E}-10$ \\
\hline
\end{tabular}

Note.

${ }^{\mathrm{a}}$ Convolved with a beam of 18.8 arcsec.

in the Orion region, where the specific angular momentum was statistically investigated by Tatematsu et al. (2016), and also because this core shows a clear velocity gradient, we investigate its properties in detail. It is well known that the specific angular momentum (angular momentum per unit mass) $J / M$ is roughly proportional to $R^{1.6}$ for molecular clouds and their cores having sizes of 0.1 to $30 \mathrm{pc}$ in general (Goldsmith \& Arquilla 1985; Goodman et al. 1993; Bodenheimer 1995). We compare the radius, velocity gradient, and the specific angular momentum among these emission lines. The beam-corrected half-intensity radius $R$ is $0.26,0.16$, and $0.058 \mathrm{pc}$, the velocity gradient is $3.9,2.2$, and $0.23 \mathrm{~km} \mathrm{~s}^{-1}$, and $J / M$ is $1.3 \times 10^{23}$, $4.5 \times 10^{22}$, and $1.7 \times 10^{21} \mathrm{~cm}^{2} \mathrm{~s}^{-1}$, respectively, in ${ }^{13} \mathrm{CO}$ $J=1-0$ and 2-1 (Liu et al. 2016) and $\mathrm{N}_{2} \mathrm{H}^{+}$(this study). Tatematsu et al. (2016) investigated the specific angular momentum $J / M$ of $\mathrm{N}_{2} \mathrm{H}^{+}$cores in the Orion $\mathrm{A}$ GMC, and compared them with cold cloud cores observed in $\mathrm{NH}_{3}$ by Goodman et al. (1993). Figure 15 plots the specific angular momentum $J / M$ of G192.32-11.88 cores observed in this study as well as Tatematsu et al. (2016), Goodman et al. (1993). $J / M$ of $\mathrm{G} 192.32-11.88$ in $\mathrm{N}_{2} \mathrm{H}^{+}$is within the range found for cores in the Orion A GMC, but is located at the high end of it. It is possible that compression by the $\mathrm{H}$ II region has resulted in relatively large $J / M$ found in the present study.

Nobeyama T70 observations were made toward G192N associated with the Class 0 like protostar. The column density ratio of $N(\mathrm{DNC})$ to $N\left(\mathrm{HN}^{13} \mathrm{C}\right)$ is 5.8 , which is larger than the value of 3.0 at L1544 (Hirota et al. 2003; Hirota \& Yamamoto 2006). Detection of $\mathrm{DNC}$ and $\mathrm{N}_{2} \mathrm{D}^{+}$indicates that this core is chemically relatively young, although the core is star-forming.

\section{8. $G 202.00+2.65$}

This clump is located in the dark cloud L1613 (Lynds 1962; Dobashi et al. 2005) and Khavtassi 308 (Khavtassi 1960). $T_{k}$ is derived to be $<10.2 \mathrm{~K}$. Figure 8 shows the continuum map. The upper limit to the kinetic temperature $(10.2 \mathrm{~K})$ was obtained from $\mathrm{NH}_{3}$ observations.

\section{9. $G 202.31-8.92$}

G202.31-8.92 is located in L1611 Lynds (1962), Dobashi et al. (2005) in the Orion Northern Filament, and close to No. 52 CO Emission Peak cataloged by Maddalena et al. (1986), which has two CO velocity components 8.8 and $11.4 \mathrm{~km} \mathrm{~s}^{-1}$. As Maddalena et al. (1986) discussed, we assume that the distance to G202.31-8.92 is similar to that of Orion A and B GMCs. Figure 9 shows the continuum map. $T_{k}$ is derived to be $<11.6 \mathrm{~K}$. Two velocity components $\left(9.2\right.$ and $\left.12.0 \mathrm{~km} \mathrm{~s}^{-1}\right)$ were detected in the ${ }^{12} \mathrm{CO}(1-0)$ and ${ }^{13} \mathrm{CO}(1-0)$ emission toward this source (Liu et al. 2012). The continuum emission shows a morphology similar to that of the $12.0 \mathrm{~km} \mathrm{~s}^{-1} \mathrm{CO}$ clump. The velocity of the $\mathrm{NH}_{3}$ emission is $11.92 \mathrm{~km} \mathrm{~s}^{-1}$, which is similar to that of the $12.0 \mathrm{~km} \mathrm{~s}^{-1} \mathrm{CO}$ clump.

The upper limit to the kinetic temperature $(11.6 \mathrm{~K})$ was obtained from $\mathrm{NH}_{3}$ observations.

Table 11

Fractional Abundance toward the T70 Position

\begin{tabular}{|c|c|c|c|c|c|c|c|}
\hline Source & $\begin{array}{c}S_{\nu}^{\text {beama }} \\
(\mathrm{mJy} / \text { beam })\end{array}$ & $\begin{array}{c}N_{\mathrm{H}_{2}} \\
\left(10^{22} \mathrm{~cm}^{-2}\right)\end{array}$ & $X(\mathrm{CCS})$ & $X\left(\mathrm{~N}_{2} \mathrm{H}^{+}\right)$ & $X\left(\mathrm{~N}_{2} \mathrm{D}^{+}\right)$ & $X\left(\mathrm{HN}^{13} \mathrm{C}\right)$ & $X(\mathrm{DNC})$ \\
\hline G089.9-01.9 & 277.7 & 2.0 & $3.3 \mathrm{E}-10$ & $2.1 \mathrm{E}-10$ & $2.9 \mathrm{E}-10$ & $7.5 \mathrm{E}-11$ & $3.8 \mathrm{E}-10$ \\
\hline G108N & 225.4 & 1.0 & $4.8 \mathrm{E}-10$ & $\ldots$ & $\ldots$ & $\ldots$ & $1.9 \mathrm{E}-10$ \\
\hline G120N & 629.3 & 2.8 & $1.3 \mathrm{E}-10$ & $3.2 \mathrm{E}-10$ & $\ldots$ & $4.3 \mathrm{E}-11$ & $7.2 \mathrm{E}-11$ \\
\hline G149.5-1.2 & 108.2 & 0.4 & $\ldots$ & $\ldots$ & $\ldots$ & $5.9 \mathrm{E}-11$ & $1.9 \mathrm{E}-10$ \\
\hline G157.6-12.2 & 162.3 & 1.1 & $5.2 \mathrm{E}-10$ & $1.2 \mathrm{E}-09$ & $5.2 \mathrm{E}-10$ & $1.2 \mathrm{E}-10$ & $1.2 \mathrm{E}-09$ \\
\hline G192.33-11.88 & 623.9 & 3.5 & $\ldots$ & $4.2 \mathrm{E}-10$ & $4.7 \mathrm{E}-11$ & $2.2 \mathrm{E}-11$ & $1.4 \mathrm{E}-10$ \\
\hline G204.4-11.3 & 1128.8 & 9.3 & $1.3 \mathrm{E}-10$ & $2.1 \mathrm{E}-10$ & $4.6 \mathrm{E}-11$ & $3.8 \mathrm{E}-11$ & $\ldots$ \\
\hline G224.4-0.6 & 88.4 & 0.3 & $\cdots$ & $\cdots$ & $\cdots$ & $\cdots$ & $\cdots$ \\
\hline Median & 251.6 & 1.6 & $3.3 \mathrm{E}-10$ & $3.2 \mathrm{E}-10$ & $1.7 \mathrm{E}-10$ & $5.1 \mathrm{E}-11$ & $1.9 \mathrm{E}-10$ \\
\hline
\end{tabular}

Note.

${ }^{\mathrm{a}}$ Convolved with a beam of 18.8 arcsec. 

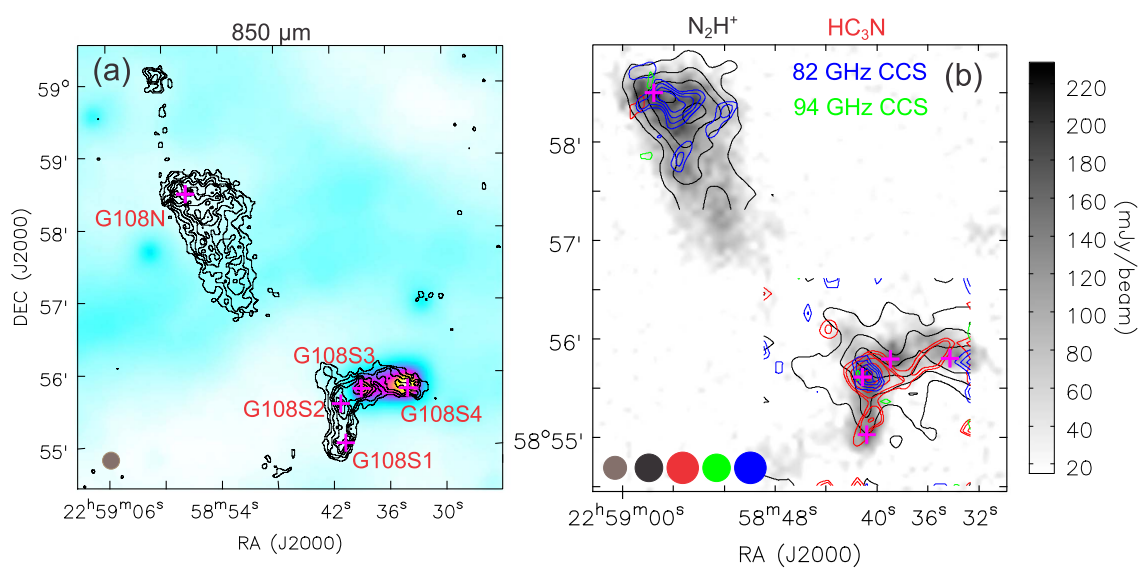

Figure 2. Same as Figure 1 but for G108.8-00.8. (a) The $850 \mu$ m continuum (contours) levels are $[0.2,0.3,0.4,0.5,0.6,0.7,0.8,0.9] \times 231.9 \mathrm{mJy} /$ beam. (b) For G108N, the $\mathrm{N}_{2} \mathrm{H}^{+}$intensity (black contours) is calculated from a velocity range of -51.5 to $-47.5 \mathrm{~km} \mathrm{~s}^{-1}$ with levels of $[3,6,9,12,15] \times 0.088 \mathrm{~K} \mathrm{~km} \mathrm{~s}{ }^{-1}(1 \sigma)$. For $\mathrm{G} 108 \mathrm{~S}$, the $\mathrm{N}_{2} \mathrm{H}^{+}$intensity (black contours) is calculated from a velocity range of -52.5 to $-48.5 \mathrm{~km} \mathrm{~s}^{-1}$ with levels of $[3,6,9,12,15] \times 0.066 \mathrm{~K} \mathrm{~km} \mathrm{~s}{ }^{-1}(1 \sigma)$. For $\mathrm{G} 108 \mathrm{~N}$, the $\mathrm{HC}_{3} \mathrm{~N}$ intensity (red) is calculated from a velocity range of -51.5 to $-47.5 \mathrm{~km} \mathrm{~s}^{-1}$ with levels of $2.5 \times 0.078 \mathrm{~K} \mathrm{~km} \mathrm{~s}(1 \sigma)$. For G108S, the $\mathrm{HC} \mathrm{C}_{3} \mathrm{~N}$ intensity (red) is calculated from a velocity range of -52.5 to $-48.5 \mathrm{~km} \mathrm{~s}^{-1}$ with levels of $[2.5,3,4,5,6] \times 0.064 \mathrm{~K} \mathrm{~km} \mathrm{~s}{ }^{-1}(1 \sigma)$. For G108N, the $82 \mathrm{GHz}$ CCS intensity (blue) is calculated from a velocity range of -51.5 to $-47.5 \mathrm{~km} \mathrm{~s}^{-1}$ with levels of $[2.5,3,3.5,4,4.5] \times 0.080 \mathrm{~K} \mathrm{~km} \mathrm{~s}{ }^{-1}(1 \sigma)$. For G108S, the $82 \mathrm{GHz}$ CCS intensity (blue) is calculated from a velocity range of -52.5 to $-48.5 \mathrm{~km} \mathrm{~s}^{-1}$ with levels of $[2.5,3,3.5,4,4.5] \times 0.078 \mathrm{~K} \mathrm{~km} \mathrm{~s}^{-1}(1 \sigma)$. For G108N, the $94 \mathrm{GHz}$ CCS intensity (green) is calculated from a velocity range of -51.5 to $-47.5 \mathrm{~km} \mathrm{~s}^{-1}$ with levels of $2.5 \times 0.11 \mathrm{~K} \mathrm{~km} \mathrm{~s}^{-1}(1 \sigma)$. For G108S, the $94 \mathrm{GHz}$ CCS intensity (green) is calculated from a velocity range of -52.5 to $-48.5 \mathrm{~km} \mathrm{~s}^{-1}$ with levels of $2.5 \times 0.076 \mathrm{~K} \mathrm{~km} \mathrm{~s}^{-1}(1 \sigma)$.
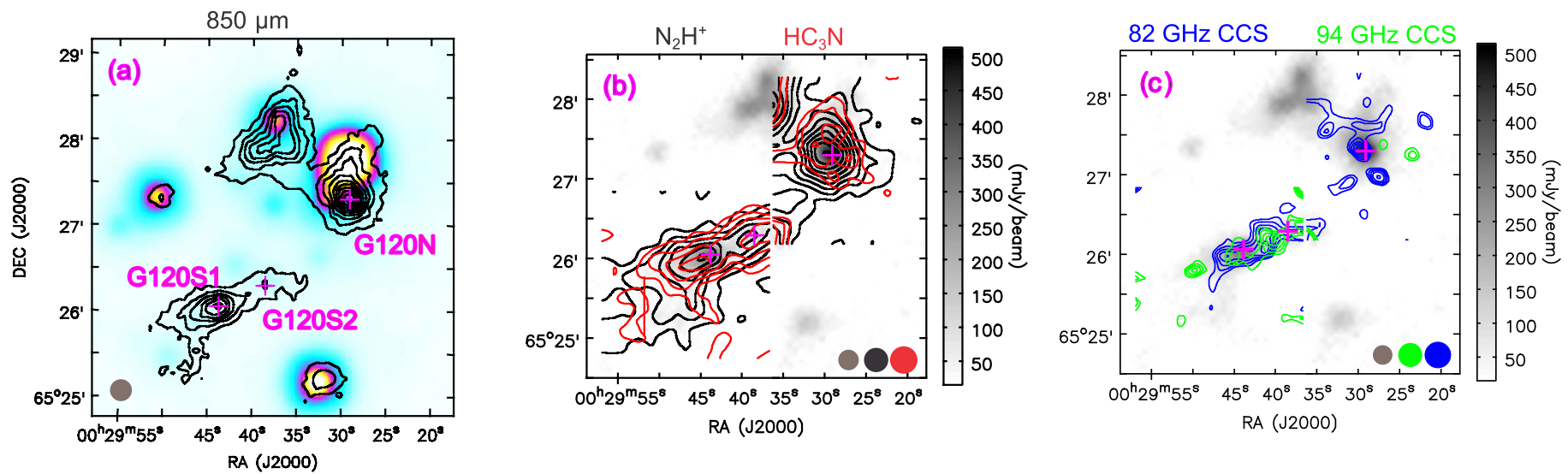

Figure 3. Same as Figure 1 but for G120.7+2.7. (a) The $850 \mu$ m continuum (contours) levels are $[0.1,0.2,0.3,0.4,0.5,0.6,0.7,0.8,0.9] \times 514.4 \mathrm{mJy} /$ beam. (b) For G120N, the $\mathrm{N}_{2} \mathrm{H}^{+}$intensity (black contours) is calculated from a velocity range of -19.5 to $-15.5 \mathrm{~km} \mathrm{~s}^{-1}$ with levels of $[3,6,9,12,15,18,21,24,27] \times 0.098 \mathrm{~K} \mathrm{~km}$ $\mathrm{s}^{-1}(1 \sigma)$. For G120S, the $\mathrm{N}_{2} \mathrm{H}^{+}$intensity (black contours) is calculated from a velocity range of -19.5 to $-16.5 \mathrm{~km} \mathrm{~s}^{-1}$ with levels of $[2.5,3,3.5,4,4.5,5,6$, 7] $\times 0.099 \mathrm{~K} \mathrm{~km} \mathrm{~s}^{-1}(1 \sigma)$. For G120N, the $\mathrm{HC}_{3} \mathrm{~N}$ intensity (red) is calculated from a velocity range of -19.5 to $-15.5 \mathrm{~km} \mathrm{~s}^{-1}$ with levels of $[3,4.5,6] \times 0.090 \mathrm{~K}$ km $\mathrm{s}^{-1}(1 \sigma)$. For G120S, the $\mathrm{HC}_{3} \mathrm{~N}$ intensity (red) is calculated from a velocity range of -19.5 to $-16.5 \mathrm{~km} \mathrm{~s}^{-1}$ with levels of $[3,6,9,12] \times 0.062 \mathrm{~K} \mathrm{~km} \mathrm{~s}{ }^{-1}(1 \sigma)$. (c) For G120N, the $82 \mathrm{GHz} C \mathrm{CS}$ intensity (blue) is calculated from a velocity range of -19.5 to $-15.5 \mathrm{~km} \mathrm{~s}^{-1}$ with levels of $[2.5,3,3.5,4,4.5] \times 0.1 \mathrm{~K} \mathrm{~km} \mathrm{~s}{ }^{-1}(1 \sigma)$. For G120S, the $82 \mathrm{GHz} C C S$ intensity (blue) is calculated from a velocity range of -19.5 to $-16.5 \mathrm{~km} \mathrm{~s}^{-1}$ with levels of $[3,3.5,4,4.5,5] \times 0.080 \mathrm{~K} \mathrm{~km} \mathrm{~s}{ }^{-1}(1 \sigma)$. For G120N, the $94 \mathrm{GHz}$ CCS intensity (green) is calculated from a velocity range of -19.5 to $-15.5 \mathrm{~km} \mathrm{~s}^{-1}$ with levels of $[2.5,3,3.5] \times 0.11 \mathrm{~K} \mathrm{~km} \mathrm{~s}{ }^{-1}(1 \sigma)$. For G120S, the $94 \mathrm{GHz} \mathrm{CCS}$ intensity (green) is calculated from a velocity range of -19.5 to $-16.5 \mathrm{~km} \mathrm{~s}^{-1}$ with levels of $[3,3.5,4,4.5,5] \times 0.085 \mathrm{~K} \mathrm{~km} \mathrm{~s}^{-1}(1 \sigma)$.

\subsection{G204.4-11.3}

G204.4-11.3 is located in L1621 Lynds (1962), Dobashi et al. (2005) and the Orion B GMC, and close to No.37 CO Emission Peak cataloged by Lynds (1962), Maddalena et al. (1986), which has two CO velocity components 8.6 and 10.9 $\mathrm{km} \mathrm{s}^{-1}$. It is located near the edge of the dark cloud Khavtassi 311 (Khavtassi 1960). As Maddalena et al. (1986) discussed, we assume that the distance to G204.4-11.3 is similar to that of Orion A and B GMCs. Figure 10 shows maps toward G20411.3. The $\mathrm{N}_{2} \mathrm{H}^{+}$emission shows a peak toward the starless peak G204NE in the $850 \mu \mathrm{m}$ continuum, but with a marginal offset of $\sim 10^{\prime \prime}$. The $\mathrm{HC}_{3} \mathrm{~N}$ is more extended than $850 \mu \mathrm{m}$ (and $\mathrm{N}_{2} \mathrm{H}^{+}$). Toward G204NE (virtually identical to the T70 position), we detected the $82 \mathrm{GHz}$ CCS emission (Tables 4 and 5), but the emission is too weak and narrow to draw a reliable map. In the single pointing observations toward G204NE, we have detected both DNC and $\mathrm{N}_{2} \mathrm{D}^{+}$. The column density ratio of $N(\mathrm{DNC})$ to $N\left(\mathrm{HN}^{13} \mathrm{C}\right)$ is 15.0 , which is much larger than the value of 3.0 at L1544 (Hirota et al. 2003; Hirota $\&$ Yamamoto 2006). The high deuterium fraction ratio implies that G204NE is a starless core on the verge of star formation. Figure 16 shows the moment 1 radial velocity map toward G204-11.3 in $\mathrm{N}_{2} \mathrm{H}^{+}$. We do not see a prominent velocity gradient $\left(<0.5 \mathrm{~km} \mathrm{~s}^{-1} \operatorname{arcmin}^{-1}\right.$ or $\left.<4 \mathrm{~km} \mathrm{~s}^{-1} \mathrm{pc}^{-1}\right)$.

\subsection{G207.3-19.8}

G207.3-19.8 is located in the Orion A GMC, and close to the No. $19 \mathrm{CO}$ emission peak identified by Maddalena et al. (1986). G207N is associated with the Herbig-Haro object HH58, which is a Class 0 like source. G207S is starless. 

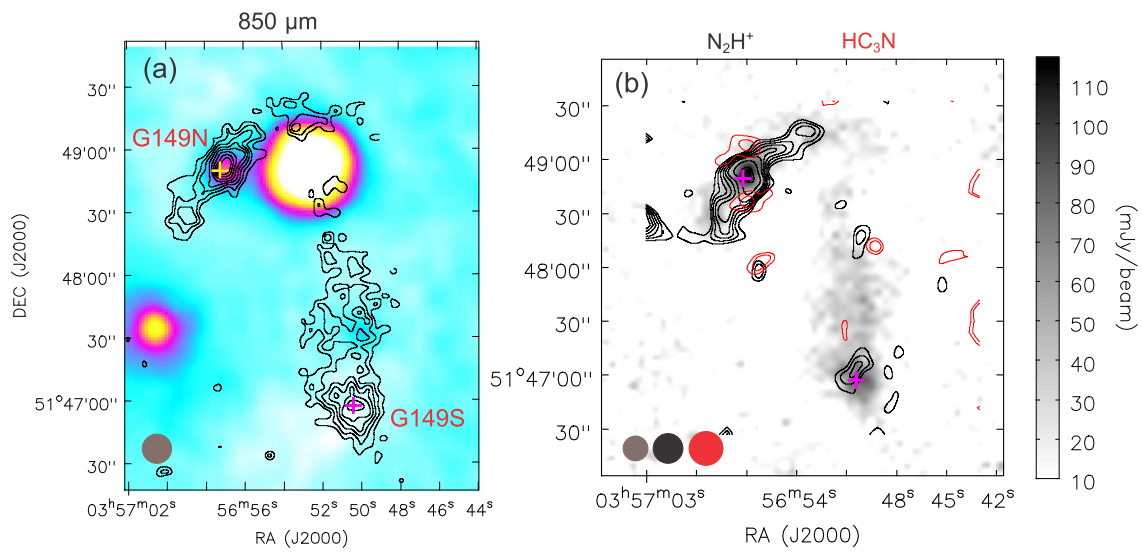

Figure 4. Same as Figure 1 but for G149.5-1.2. (a) The $850 \mu \mathrm{m}$ continuum (contours) levels are [0.2, 0.3, 0.4, 0.5, 0.6, 0.7, 0.8, 0.9] $\times 117.2 \mathrm{mJy} /$ beam. (b) The $\mathrm{N}_{2} \mathrm{H}^{+}$intensity (black contours) is calculated from a velocity range of -8.5 to $-5.5 \mathrm{~km} \mathrm{~s}^{-1}$ with levels of $[2.5,3,3.5,4,4.5,5,6,7] \times 0.099 \mathrm{~K} \mathrm{~km} \mathrm{~s}{ }^{-1}(1 \sigma)$. The $\mathrm{HC}_{3} \mathrm{~N}$ intensity (red) is calculated for a velocity range of -8.5 to $-5.5 \mathrm{~km} \mathrm{~s}^{-1}$ with levels of $[2.5,3] \times 0.080 \mathrm{~K} \mathrm{~km} \mathrm{~s}^{-1}(1 \sigma)$.
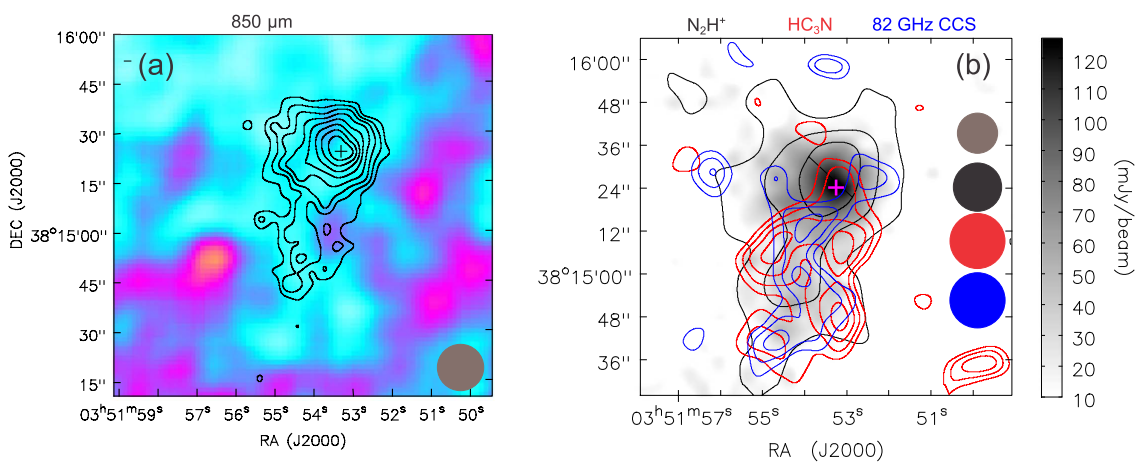

Figure 5. Same as Figure 1 but for G157.6-12.2. (a) The $850 \mu$ m continuum (contours) levels are $[0.2,0.3,0.4,0.5,0.6,0.7,0.8,0.9] \times 126.8 \mathrm{mJy} /$ beam. (b) The $\mathrm{N}_{2} \mathrm{H}^{+}$intensity (black contours) is calculated from a velocity range of -8.5 to $-6.5 \mathrm{~km} \mathrm{~s}^{-1}$ with levels of $[3,6,9] \times 0.078 \mathrm{~K} \mathrm{~km} \mathrm{~s}^{-1}(1 \sigma)$. The $\mathrm{HC}_{3} \mathrm{~N}$ intensity $(\mathrm{red})$ is calculated from a velocity range of -8.5 to $-6.5 \mathrm{~km} \mathrm{~s}^{-1}$ with levels of $[2.5,3,3.5,4] \times 0.071 \mathrm{~K} \mathrm{~km} \mathrm{~s}^{-1}(1 \sigma)$. The $82 \mathrm{GHz}$ CCS intensity (blue) is calculated from a velocity range of -8.5 to $-6.5 \mathrm{~km} \mathrm{~s}^{-1}$ with levels of $[2.5,3,3.5] \times 0.086 \mathrm{~K} \mathrm{~km} \mathrm{~s}^{-1}(1 \sigma)$.
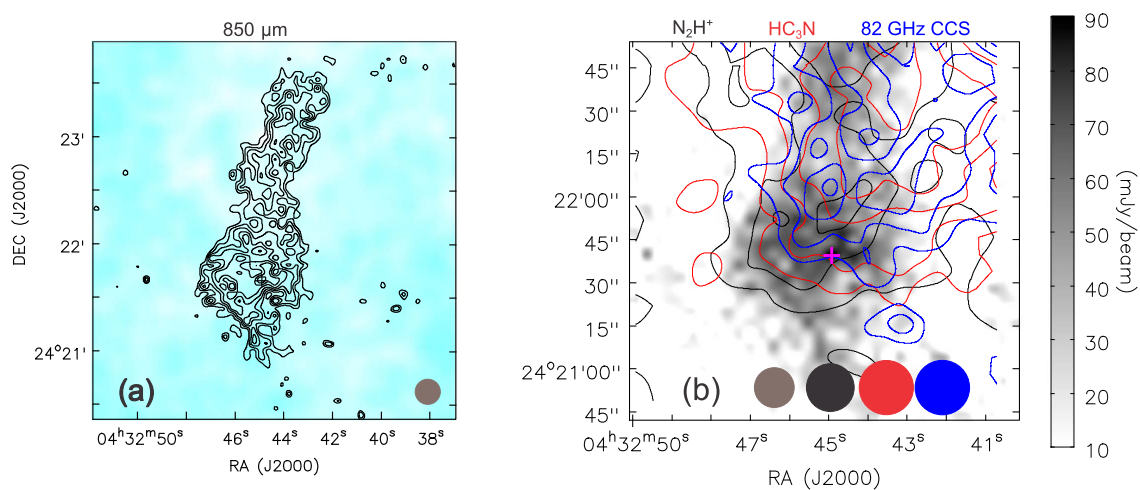

Figure 6. Same as Figure 1 but for G174.0-15.8. (a) The $850 \mu \mathrm{m}$ continuum (contours) levels are $[0.3,0.4,0.5,0.6,0.7,0.8,0.9] \times 90.3 \mathrm{mJy} / \mathrm{beam}$. (b) Black contours represent intensity integrated for a velocity range of 5.5 to $7.5 \mathrm{~km} \mathrm{~s}^{-1}$ with levels of $[3,4.5,6] \times 0.065 \mathrm{~K} \mathrm{~km} \mathrm{~s}^{-1}(1 \sigma)$. The $\mathrm{HC}_{3} \mathrm{~N}$ intensity (red) is calculated from a velocity range of 5.5 to $7.5 \mathrm{~km} \mathrm{~s}^{-1}$ with levels of $[3,6,9,12] \times 0.052 \mathrm{~K} \mathrm{~km} \mathrm{~s}^{-1}(1 \sigma)$. The $82 \mathrm{GHz} \mathrm{CCS}$ intensity (blue) is calculated from a velocity range of 5.5 to $7.5 \mathrm{~km} \mathrm{~s}^{-1}$ with levels of $[3,4.5,6] \times 0.065 \mathrm{~K} \mathrm{~km} \mathrm{~s}^{-1}(1 \sigma)$.

Figure 11 shows maps toward G207N. The $\mathrm{N}_{2} \mathrm{H}^{+}$emission is well correlated with the $850 \mu \mathrm{m}$ distribution. The $\mathrm{N}_{2} \mathrm{H}^{+}$ emission shows a peak toward G207N1 associated with $\mathrm{HH} 58$. The $\mathrm{HC}_{3} \mathrm{~N}$ also shows a peak toward G207N1. Weak emission is observed also toward G207N2, G207N3, G207N4, and G207N5 in $\mathrm{N}_{2} \mathrm{H}^{+}$, but not in $\mathrm{HC}_{3} \mathrm{~N}$. We have not detected either the $82 \mathrm{GHz}$ or $94 \mathrm{GHz} \mathrm{CCS}$ emission. In single pointing observation toward the T70 position, which was mistakenly set north of $\mathrm{G} 207 \mathrm{~N}$, we have not detected either DNC or $\mathrm{N}_{2} \mathrm{D}^{+}$.
Figure 12 shows the $\mathrm{N}_{2} \mathrm{H}^{+}$map toward G207S. The $\mathrm{N}_{2} \mathrm{H}^{+}$ emission is more or less correlated with the $850 \mu \mathrm{m}$ distribution, but their peak positions do not coincide completely with each other. We have not detected either the $\mathrm{HC}_{3} \mathrm{~N}$, $82 \mathrm{GHz}$ or $94 \mathrm{GHz} \mathrm{CCS}$ emission.

\subsection{G224.4-0.6}

This clump is located in L1658 Lynds (1962), Dobashi et al. (2005), the Orion Southern Filament (Genzel \& Stutzki 1989), 

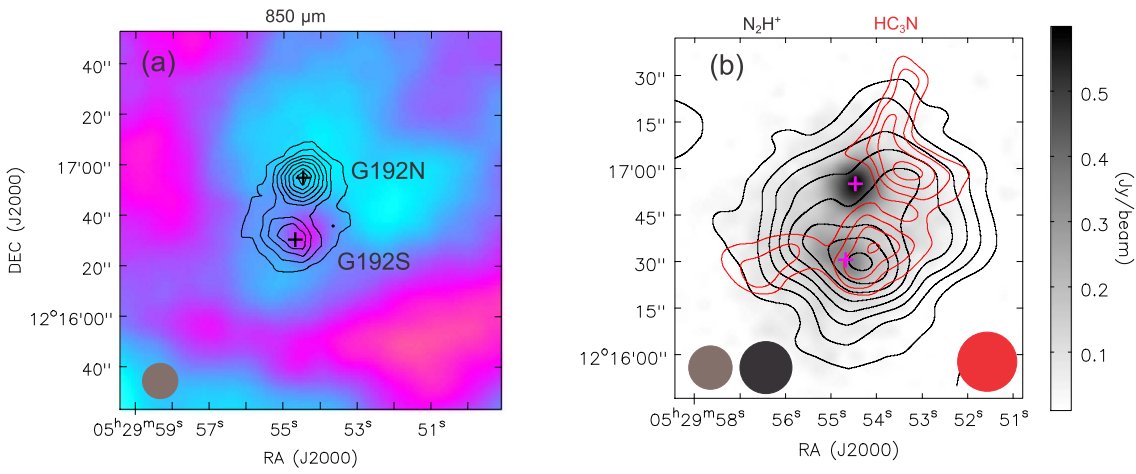

Figure 7. Same as Figure 1 but for G192.32-11.88. (a) The $850 \mu$ m continuum (contours) levels are $[0.2,0.3,0.4,0.5,0.6,0.7,0.8,0.9] \times 600 \mathrm{mJy} /$ beam. (b) The $\mathrm{N}_{2} \mathrm{H}^{+}$intensity (black contours) is calculated from a velocity range of 11.5 to $13.5 \mathrm{~km} \mathrm{~s}^{-1}$ with levels of $[3,6,9,12,15,18,21,24] \times 0.081 \mathrm{~K} \mathrm{~km} \mathrm{~s}(1 \sigma)$. The $\mathrm{HC}_{3} \mathrm{~N}$ intensity (red) is calculated from a velocity range of 11.5 to $13.5 \mathrm{~km} \mathrm{~s}^{-1}$ with levels of $[3,4,5,6] \times 0.069 \mathrm{~K} \mathrm{~km} \mathrm{~s} \mathrm{k}^{-1}(1 \sigma)$.

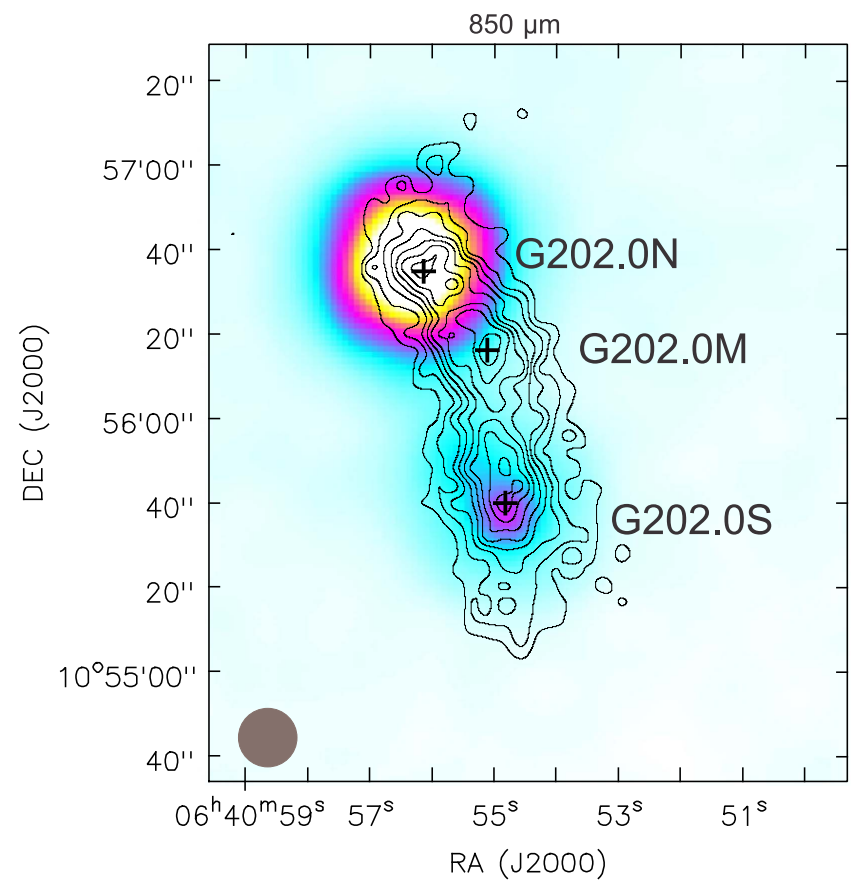

Figure 8. The $850 \mu \mathrm{m}$ continuum image (contours) obtained with JCMT/ SCUBA-2 toward G202.00+2.65 superimposed on the WISE $22 \mu \mathrm{m}$ image (color). Contour levels are $[0.2,0.3,0.4,0.5,0.6,0.7,0.8,0.9] \times 88.8$ $\mathrm{mJy} /$ beam.

CMa R1 region (Nakano et al. 1984), and CMa OB1 (Blitz 1978; Kim et al. 1996) and near the dark cloud Khavtassi 330 (Khavtassi 1960). Figure 13 shows maps toward G224.4-0.6. The $\mathrm{N}_{2} \mathrm{H}^{+}$emission is well correlated with the $850 \mu \mathrm{m}$ distribution. Both tracers have two prominent intensity peaks, i.e., G224NE and G224S. We see slight peak position offsets. The $\mathrm{HC}_{3} \mathrm{~N}$ emission is not well correlated with the $850 \mu \mathrm{m}$ distribution, and shows a stronger peak toward the southern $850 \mu \mathrm{m}$ source. The $82 \mathrm{GHz}$ and $94 \mathrm{GHz} \mathrm{CCS}$ emissions have local emission peaks between the two $850 \mu \mathrm{m}$ sources. T70 observations were carried out toward G224NE, and we detected DNC and $\mathrm{HN}^{13} \mathrm{C}$. Figure 17 shows the moment 1 radial velocity map in $\mathrm{N}_{2} \mathrm{H}^{+}$. We see a steep velocity gradient. The SE-NW gradient is about $0.9 \mathrm{~km} \mathrm{~s}^{-1}$ $\operatorname{arcmin}^{-1}$ or $3 \mathrm{~km} \mathrm{~s}^{-1} \mathrm{pc}^{-1}$.

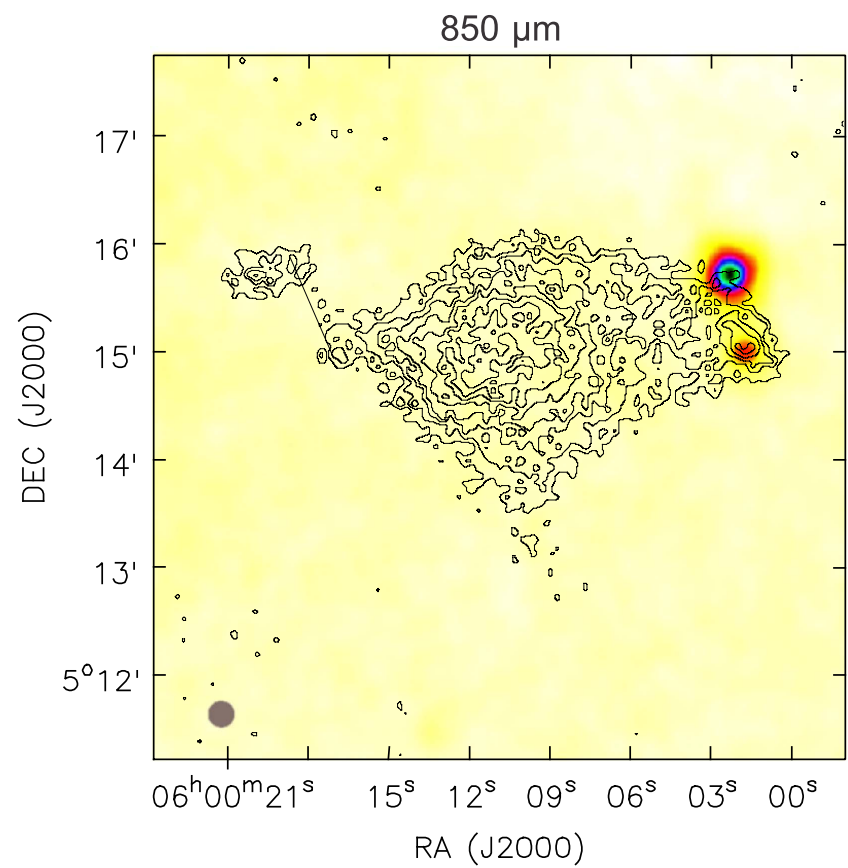

Figure 9. Same as Figure 8 but for G202.31-8.92. Contour levels are [0.2, 0.3, $0.4,0.5,0.6,0.7,0.8,0.9] \times 231.2 \mathrm{mJy} /$ beam .

\section{Discussion}

\subsection{Column Density Ratios}

Hirota \& Yamamoto (2006) indicated the evolutionary sequence of starless cores by using column density ratios such as $N(\mathrm{DNC}) / N\left(\mathrm{HN}^{13} \mathrm{C}\right)$. It seems that $N(\mathrm{DNC}) / N\left(\mathrm{HN}^{13} \mathrm{C}\right)$ in starless cores increases with core evolution $(<0.66$ to 3$)$. Using their data, we can show that $N\left(\mathrm{~N}_{2} \mathrm{H}^{+}\right) / N(\mathrm{CCS})$ is $\lesssim 0.12$ for young starless cores (L1495B,L1521B, L1521E, TMC-1, and L492). According to Tatematsu et al. (2014b), $N\left(\mathrm{~N}_{2} \mathrm{H}^{+}\right) / N$ (CCS) is usually $\lesssim 2-3$ for starless cores, but can reach $\sim 2-3$ for evolved starless cores. Star-forming cores generally give $N$ $\left(\mathrm{N}_{2} \mathrm{H}^{+}\right) / N(\mathrm{CCS}) \gtrsim 2-3$.

In the present observations, $N\left(\mathrm{~N}_{2} \mathrm{H}^{+}\right) / N(\mathrm{CCS})$ ranges from 0.4 to 3.7 , and $N(\mathrm{DNC}) / N\left(\mathrm{HN}^{13} \mathrm{C}\right)$ ranges from 1.7 to 10 . These results suggest that our Planck cold clumps consist of various evolutionary stages including relatively young starless cores and those on the verge of star formation. In the present observations, $N\left(\mathrm{~N}_{2} \mathrm{D}^{+}\right) / N\left(\mathrm{~N}_{2} \mathrm{H}^{+}\right)$ranges from 0.1 to 1.4. 

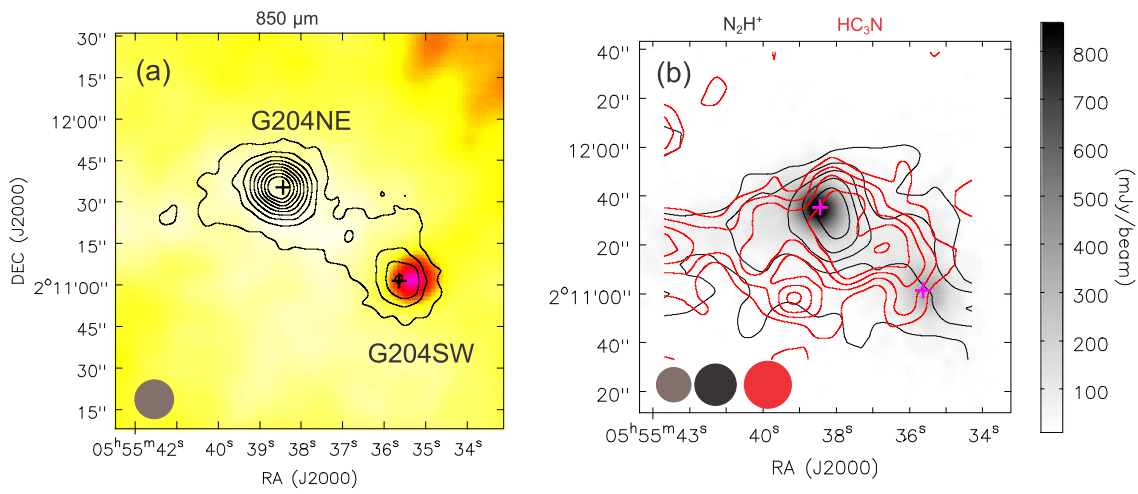

Figure 10. Same as Figure 1 but for G204.4-11.3. (a) The $850 \mu$ m continuum (contours) levels are $[0.1,0.2,0.3,0.4,0.5,0.6,0.7,0.8,0.9] \times 861.5 \mathrm{mJy} /$ beam. (b) The $\mathrm{N}_{2} \mathrm{H}^{+}$intensity (black contours) is calculated from a velocity range of 0.5 to $2.5 \mathrm{~km} \mathrm{~s}^{-1}$ with levels of $[3,6,9,12,15] \times 0.11 \mathrm{~K} \mathrm{~km} \mathrm{~s}^{-1}(1 \sigma)$. The $\mathrm{HC}_{3} \mathrm{~N}$ intensity (red) is calculated for intensity integrated for a velocity range of 0.5 to $2.5 \mathrm{~km} \mathrm{~s}^{-1}$ with levels of $[3,4.5,6,7.5,10] \times 0.086 \mathrm{~K} \mathrm{~km} \mathrm{~s}{ }^{-1}(1 \sigma)$.
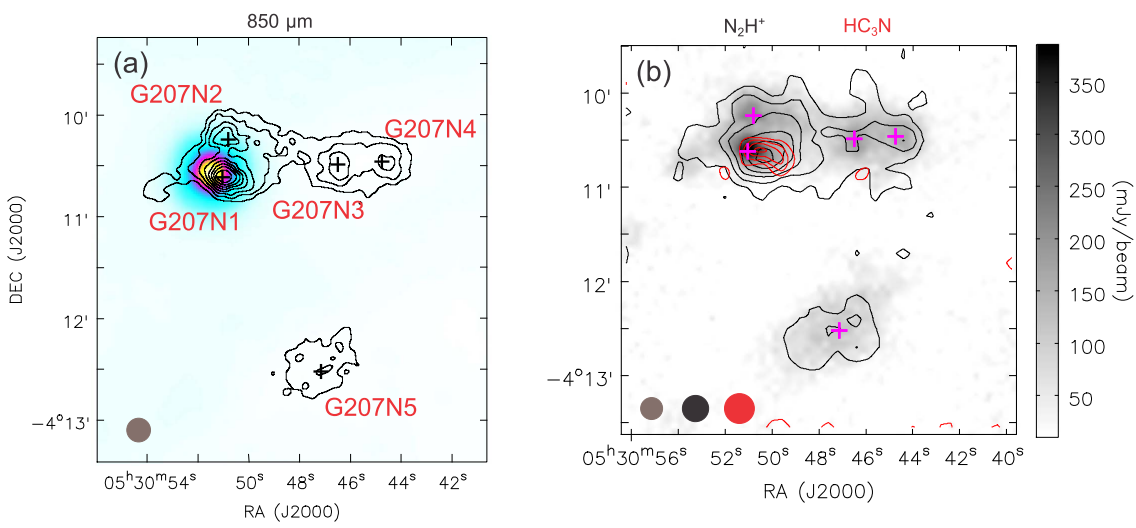

Figure 11. Same as Figure 1 but for G207N. (a) The $850 \mu$ m continuum (contours) levels are $[0.1,0.2,0.3,0.4,0.5,0.6,0.7,0.8,0.9] \times 386.4 \mathrm{mJy} /$ beam. (b) The $\mathrm{N}_{2} \mathrm{H}^{+}$intensity (black contours) is calculated from a velocity range of 9.5 to $12.5 \mathrm{~km} \mathrm{~s}^{-1}$ with levels of $[3,6,9,12,15,18] \times 0.13 \mathrm{~K} \mathrm{~km} \mathrm{~s}(1 \sigma)$. The $\mathrm{HC} \mathrm{N}_{3} \mathrm{~N}$ intensity (red) is calculated for intensity integrated for a velocity range of 9.5 to $12.5 \mathrm{~km} \mathrm{~s}^{-1}$ with levels of $[3,4.5,6,7.5] \times 0.11 \mathrm{~K} \mathrm{~km} \mathrm{~s}^{-1}(1 \sigma)$.
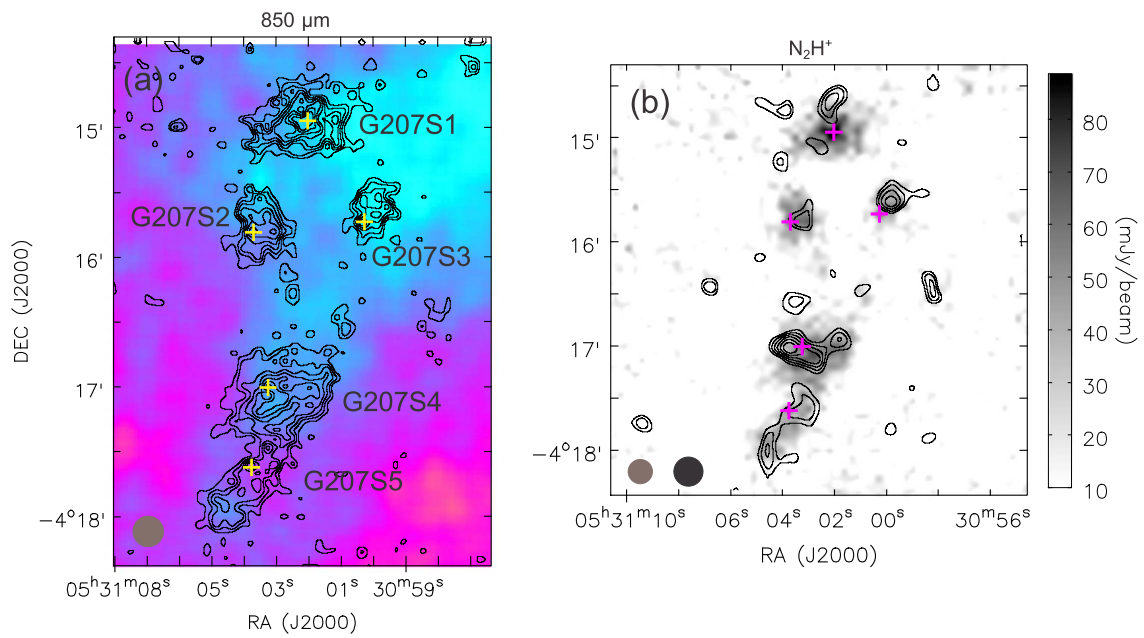

Figure 12. Same as Figure 1 but for G207S. (a)The $850 \mu \mathrm{m}$ continuum (contours) levels are $[0.2,0.3,0.4,0.5,0.6,0.7,0.8,0.9] \times 88.8 \mathrm{mJy} /$ beam. (b) The $\mathrm{N}_{2} \mathrm{H}^{+}$ intensity (black contours) is calculated from a velocity range of 11.5 to $12.5 \mathrm{~km} \mathrm{~s}^{-1}$ with levels of $[2.5,3,3.5,4,4.5,5] \times 0.083 \mathrm{~K} \mathrm{~km} \mathrm{~s}{ }^{-1}(1 \sigma)$.

Fontani et al. (2006) and Chen et al. (2011) studied the column density ratio of $\mathrm{N}_{2} \mathrm{D}^{+}$to $\mathrm{N}_{2} \mathrm{H}^{+}$(they call this the deuterium fractionation $D_{\text {frac }}$ ) toward massive protostellar cores, and compared it with those of low-mass prestellar cores by Crapsi et al. (2005). $N\left(\mathrm{~N}_{2} \mathrm{D}^{+}\right) / N\left(\mathrm{~N}_{2} \mathrm{H}^{+}\right)$is of order $10^{-1}$ in low-mass prestellar cores (Crapsi et al. 2005), and of order $10^{-2}$ in massive protostellar IRAS cores (Fontani et al. 2006). The ratio is estimated to be 0.35 and 0.08 at typical cold clouds, L134N and TMC-1N, respectively (Tiné et al. 2000). Our $\mathrm{N}_{2} \mathrm{D}^{+}$ detected cores have larger $N\left(\mathrm{~N}_{2} \mathrm{D}^{+}\right) / N\left(\mathrm{~N}_{2} \mathrm{H}^{+}\right)$values than the massive protostellar IRAS cores of Fontani et al. (2006).

In G149.5-1.2 (contains a WISE source), G192N (contains a Class 0), G207N (contains HH58), and G207S, we did not detect the $82 \mathrm{GHz}$ CCS emission over the OTF map regions. The $\mathrm{H}_{2}$ column density of our sources are $3 \times 10^{21} \mathrm{~cm}^{-2}$ or higher (Tables 10 and 11). In G149.5-1.2, the $\mathrm{H}_{2}$ column 

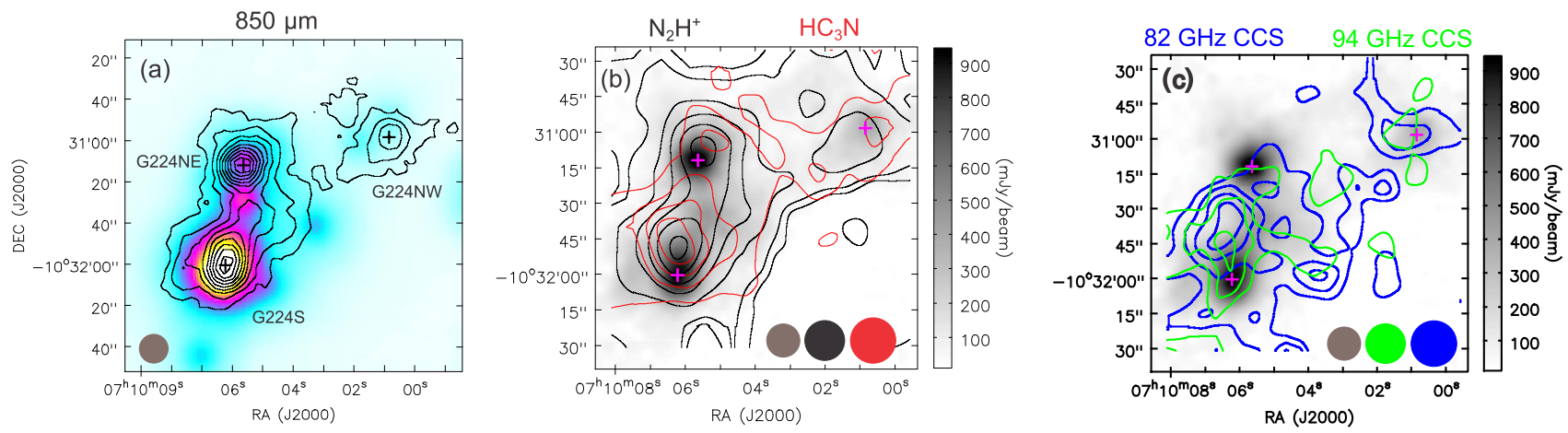

Figure 13. Same as Figure 1 but for G224.4-0.6. (a) The $850 \mu \mathrm{m}$ continuum (contours) levels are $[0.1,0.2,0.3,0.4,0.5,0.6,0.7,0.8,0.9] \times 946.5 \mathrm{mJy} /$ beam. (b) The $\mathrm{N}_{2} \mathrm{H}^{+}$intensity (black contours) is calculated from a velocity range of 12.5 to $17.5 \mathrm{~km} \mathrm{~s}^{-1}$ with levels of $[3,5,10,15,20,25,30] \times 0.15 \mathrm{~K} \mathrm{~km} \mathrm{~s}{ }^{-1}(1 \sigma)$. The $\mathrm{HC}_{3} \mathrm{~N}$ intensity (red) is calculated from a velocity range of 12.5 to $17.5 \mathrm{~km} \mathrm{~s}^{-1}$ with levels of $[3,6,9,12] \times 0.12 \mathrm{~K} \mathrm{~km} \mathrm{~s}^{-1}(1 \sigma)$. (c) The $82 \mathrm{GHz}$ CCS intensity (blue) is calculated for a velocity range of 12.5 to $17.5 \mathrm{~km} \mathrm{~s}^{-1}$ with levels of $[3,4.5,6,7.5] \times 0.13 \mathrm{~K} \mathrm{~km} \mathrm{~s}^{-1}$. The $94 \mathrm{GHz}$ CCS intensity (green) is calculated from a velocity range of 12.5 to $17.5 \mathrm{~km} \mathrm{~s}^{-1}$ with levels of $[3,4.5,6] \times 0.14 \mathrm{~K} \mathrm{~km} \mathrm{~s}^{-1}(1 \sigma)$.

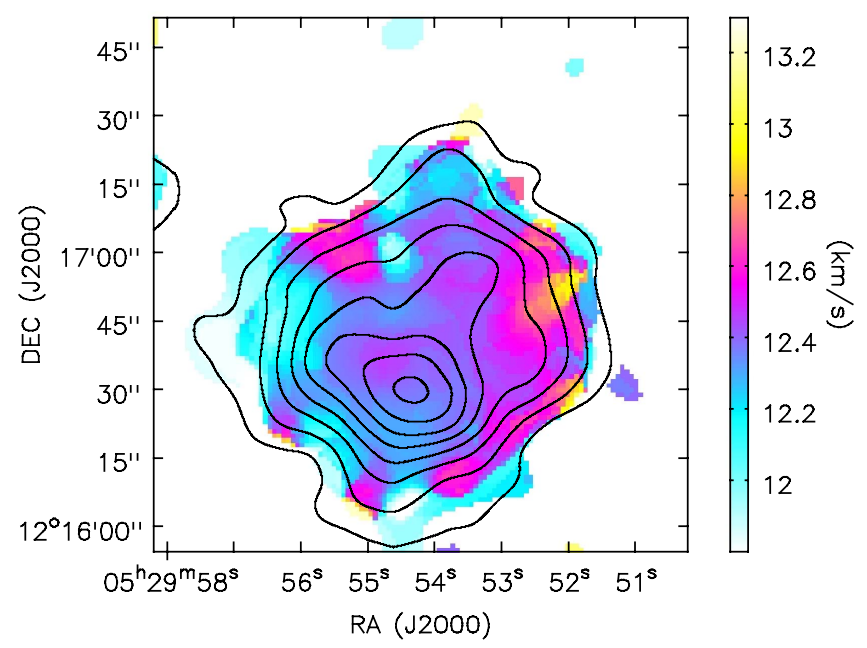

Figure 14. The intensity-weighted radial velocity (moment 1) map (color) toward G192.32-11.88 in the $\mathrm{N}_{2} \mathrm{H}^{+} J=1-0 F_{1}=2-1$ main hyperfine emission group $F_{1}=2-1 \quad(F=1-0,2-1$, and $3-2)$ overlaid on the integrated intensity (moment 0 ) contour map. The emission less than $2 \sigma$ is ignored in the moment 1 calculation. The contour levels are the same as those in Figure 7.

density is lower than $1 \times 10^{22} \mathrm{~cm}^{-2}$. It is possible that a low column density is the reason for non-detection of CCS. The $\mathrm{H}_{2}$ column density toward G192N (contains Class 0) is as high as $6 \times 10^{22} \mathrm{~cm}^{-2}$, so non-detection of CCS probably means that the gas is chemically evolved. In G108S, the $\mathrm{H}_{2}$ column density is as low as $\sim 1 \times 10^{22} \mathrm{~cm}^{-2}$, but we detected the $82 \mathrm{GHz}$ CCS emission. G089W (starless) should be chemically young, because $N\left(\mathrm{~N}_{2} \mathrm{H}^{+}\right) / N(\mathrm{CCS})$ is as small as 0.7 and $N$ $\left(\mathrm{N}_{2} \mathrm{D}^{+}\right) / N\left(\mathrm{~N}_{2} \mathrm{H}^{+}\right)$is as high as 1.4. G157.6-12.2 (starless) is young, because $N(\mathrm{DNC}) / N\left(\mathrm{HN}^{13} \mathrm{C}\right)$ is as high as 10.4 . G108N (starless) and G204NE (starless) show $N\left(\mathrm{~N}_{2} \mathrm{H}^{+}\right) / N(\mathrm{CCS})$ is $\sim 2$, which is close to the border between starless and starforming cores in Tatematsu et al. (2014b).

In the next subsection, we discuss the evolutionary stage of our Planck cold clumps on the basis of the column density ratios in Tables 8 and 9 . We assume the filling factor is unity when we estimate the column density, but it is possible that the beam filling factor is less than unity. If beam filling factors are similar between molecules, then the column density ratio will be less affected by unknown absolute values of the filling factor. Because $T_{k}$ is $\lesssim 25 \mathrm{~K}$ in general, we can use $N\left(\mathrm{~N}_{2} \mathrm{H}^{+}\right) / N$ (CCS) as a chemical evolution tracer (Tatematsu et al. 2014b).

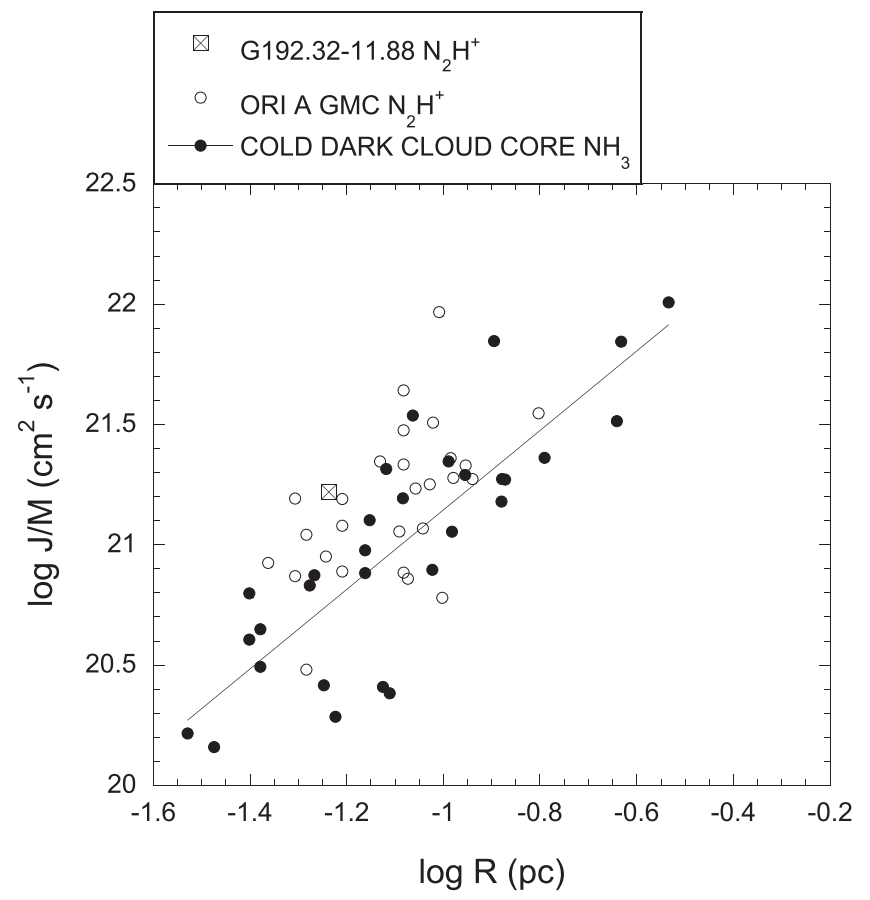

Figure 15. $J / M-R$ diagram for G192.32-11.88 in $\mathrm{N}_{2} \mathrm{H}^{+}$in this study (box with cross), Orion A GMC cores (open circles, Tatematsu et al. 2016), and cold dark cloud cores (filled circles, Goodman et al. 1993). The thin solid straight line is computed using a linear least-squares program for cold dark cloud cores.

The beam sizes for $\mathrm{CCS}$ and $\mathrm{NH}_{3}$ differ by a factor of four, and $N\left(\mathrm{NH}_{3}\right) / N(\mathrm{CCS})$ suffers from sampling/averaging over very different size scales. We thus consider $N\left(\mathrm{NH}_{3}\right) / N(\mathrm{CCS})$ much less reliable.

\subsection{Chemical Evolution Factor}

We introduce a new parameter to represent the chemical evolution by using molecular column density ratios, the chemical evolution factor (CEF). We define CEF so that starless cores have CEFs of $\sim-100$ to 0 , and star-forming cores show $\sim 0$ to 100 . Starless cores having $\mathrm{CEF} \sim 0$ are regarded as being on the verge of star formation. We use the form of $\mathrm{CEF}=\log \left([N(\mathrm{~A}) / N(\mathrm{~B})] /\left[N_{0}(\mathrm{~A}) / N_{0}(\mathrm{~B})\right]\right)^{*} d$ for the column density ratio of molecule A to molecule B. $N_{0}(\mathrm{~A}) /$ $N_{0}$ (B) is chosen to be the column density ratio at the time of star formation. For $\left(N\left(\mathrm{~N}_{2} \mathrm{H}^{+}\right) / N(\mathrm{CCS})\right.$ and $N\left(\mathrm{NH}_{3}\right) / N(\mathrm{CCS})$, 


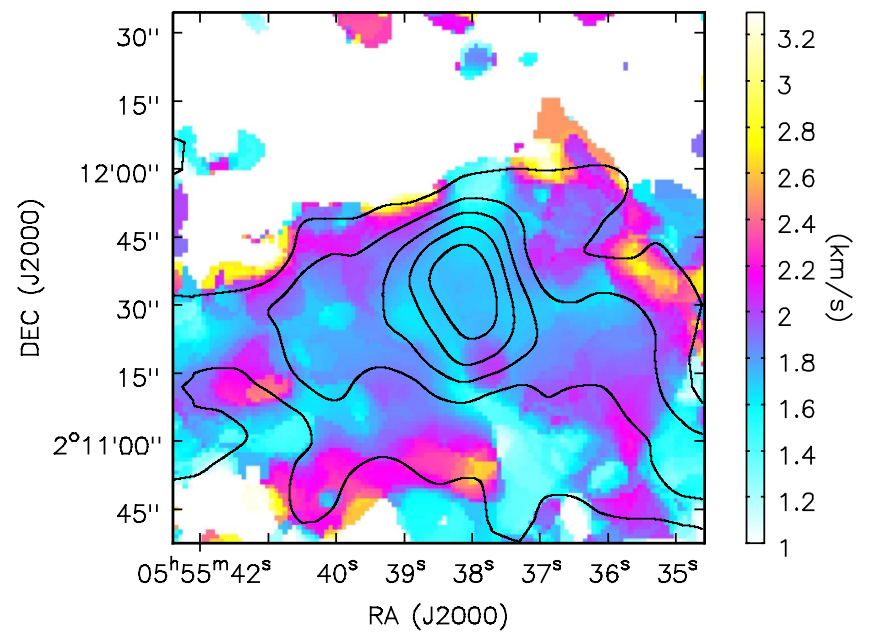

Figure 16. The radial velocity (moment 1 ) map (color) toward G204.4-11.3 in $\mathrm{N}_{2} \mathrm{H}^{+}$superimposed on the integrated intensity (moment 0 ) map. The contour levels are the same as those in Figure 12.

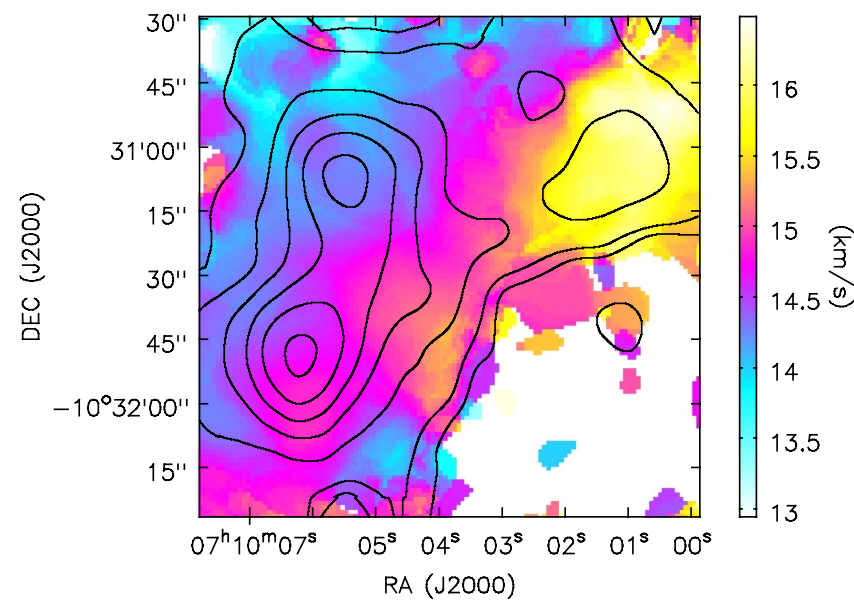

Figure 17. The radial velocity (moment 1 ) map (color) toward G224.4-0.6 in $\mathrm{N}_{2} \mathrm{H}^{+}$superimposed on the integrated intensity (moment 0 ) map. The contour levels are the same as those in Figure 16.

$N_{0}(\mathrm{~A}) / N_{0}$ (B) corresponds to the border between starless and star-forming cores. For $N(\mathrm{DNC}) / N\left(\mathrm{HN}^{13} \mathrm{C}\right)$ and $N\left(\mathrm{~N}_{2} \mathrm{D}^{+}\right) / N$ $\left(\mathrm{N}_{2} \mathrm{H}^{+}\right), N_{0}(\mathrm{~A}) / N_{0}(\mathrm{~B})$ is the highest value observed for starless cores. The factor $d$ is determined so that all starless cores range approximately from -100 to 0 . By taking into account the observational results of Suzuki et al. (1992), Crapsi et al. (2005), Hirota \& Yamamoto (2006), Tatematsu et al. (2014b), we define $\mathrm{CEF}$ as $\mathrm{CEF}=\log \left(N\left(\mathrm{~N}_{2} \mathrm{H}^{+}\right) / N(\mathrm{CCS}) / 2.5\right)^{*} 50, \log$ $\left(N(\mathrm{DNC}) / N\left(\mathrm{HN}^{13} \mathrm{C}\right) / 3\right)^{*} 120, \quad \log \left(N\left(\mathrm{~N}_{2} \mathrm{D}^{+}\right) / N\left(\mathrm{~N}_{2} \mathrm{H}^{+}\right) /\right.$ $0.3)^{*} 50$, and $\log \left(N\left(\mathrm{NH}_{3}\right) / N(\mathrm{CCS}) / 70\right)^{*} 70$, for starless cores with $T_{k} \sim 10-20 \mathrm{~K}$ at a spatial resolution of order $0.015-0.05$ pc (for $0.1 \mathrm{pc}$ sized structure "molecular cloud core"). These expressions should be only valid for the above-mentioned temperature range and spatial resolution, because we determined the CEF by using data obtained for molecular clumps or molecular cloud cores having such temperatures and observed at such spatial resolutions. The chemical reaction will depend on density, temperature, radiation strength, cosmic-ray strength, etc. The deuterium fraction will be lower for warm cores (Snell \& Wootten 1979; Wootten 1987; Schilke et al. 1992; Tatematsu et al. 2010). It seems that the deuterium fraction decreases after the onset of star formation

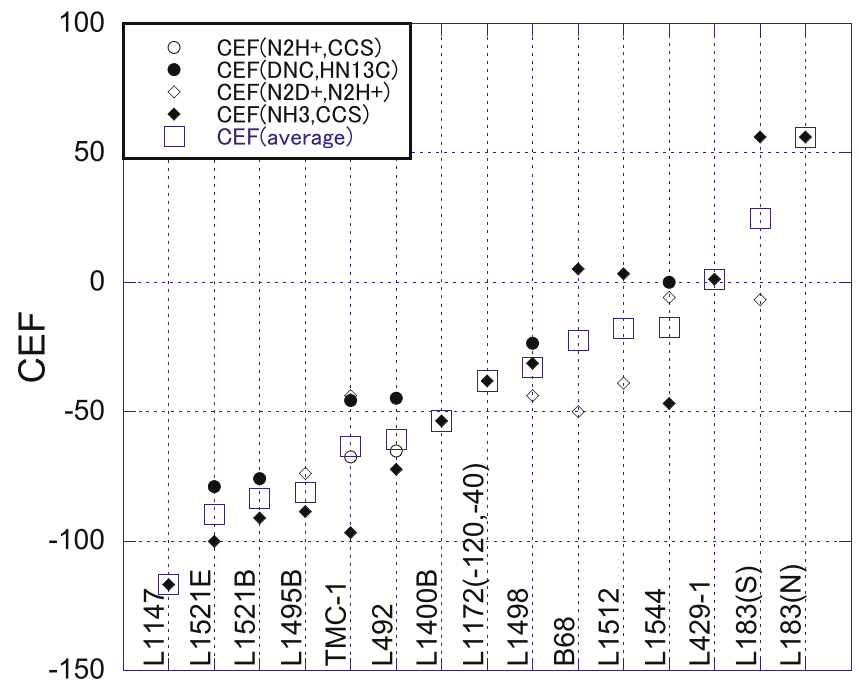

Source

Figure 18. CEF for starless sources in the literature.

Table 12

CEF Based on the $N\left(\mathrm{~N}_{2} \mathrm{H}^{+}\right) / N(\mathrm{CCS})$ Column Density Ratio toward the Starless SCUBA-2 Peak

\begin{tabular}{lc}
\hline \hline SCUBA-2 peak & CEF( $\left.\mathrm{N}_{2} \mathrm{H}^{+}, \mathrm{CCS}\right)$ \\
\hline G089E & $(8)$ \\
G089W & $(-27)$ \\
G108N & $(-19)$ \\
G108S2 & $(-2)$ \\
G120S2 & $(>19)$ \\
G120N & $(-21)$ \\
G157 & 3 \\
G174 & $-44-6$ \\
G204NE & -13 \\
G207N2 & $>-2$ \\
G207N4 & $>46$ \\
G207N5 & $>-25$ \\
G224NW & $(-83--33)$ \\
\hline
\end{tabular}

(Emprechtinger et al. 2009; Sakai et al. 2012; Fontani et al. 2014; Sakai et al. 2015). Because the nature of this decrease has not yet been fully characterized observationally, we do not use star-forming cores for CEF based on the deuterium fractionation. Figure 18 shows the resulting CEF using the data in the literature (Crapsi et al. 2005; Hirota \& Yamamoto 2006; Hirota et al. 2009).

Tables 12 and 13 and Figures 19 and 20 show the CEFs estimated in the present study. We regard cores as star-forming if the first entry of comments in Table 3 suggests a possibility of star formation (e.g., Class 0, Class I etc.). Our beam size with receivers TZ1 and T70 corresponds to 0.1 and $0.3 \mathrm{pc}$ at a distance of $700 \mathrm{pc}$ and $3.5 \mathrm{kpc}$, respectively. To see the effect of very different spatial resolution (and probably very different volume density and very different beam-filling factor), we show sources located beyond $1 \mathrm{kpc}$ in parentheses. In this paper, we treat only starless cores for CEF, because evolution of star-forming cores has not been well characterized yet. If we mistakenly identified star-forming cores as starless, we may mistakenly obtain lower CEF due to decrease in the deuterium fractionation after star formation. If this is the case, we may see 
Table 13

CEF toward the Starless T70 Position

\begin{tabular}{|c|c|c|c|c|}
\hline Source & $\mathrm{CEF}\left(\mathrm{N}_{2} \mathrm{H}^{+}, \mathrm{CCS}\right)$ & $\mathrm{CEF}\left(\mathrm{DNC}, \mathrm{HN}^{13} \mathrm{C}\right)$ & $\mathrm{CEF}\left(\mathrm{N}_{2} \mathrm{D}^{+}, \mathrm{N}_{2} \mathrm{H}^{+}\right)$ & $\mathrm{CEF}$ (average) \\
\hline G089.9-01.9 & $(-29)$ & (27) & (33) & $(10 \pm 34)$ \\
\hline G108N & $\ldots$ & $(-9)$ & $\ldots$ & $(-9)$ \\
\hline G120N & $(-1)$ & $(-30)$ & $\ldots$ & $(-16 \pm 20)$ \\
\hline G157.6-12.2 & -1 & 65 & 7 & $24 \pm 36$ \\
\hline G174.0-15.8 & $<-30$ & $\ldots$ & $\cdots$ & $\ldots$ \\
\hline G204.4-11.3 & -10 & $\ldots$ & -7 & $-8 \pm 2$ \\
\hline G224.4-0.6 & $(>3)$ & $\ldots$ & $\ldots$ & $\ldots$ \\
\hline
\end{tabular}

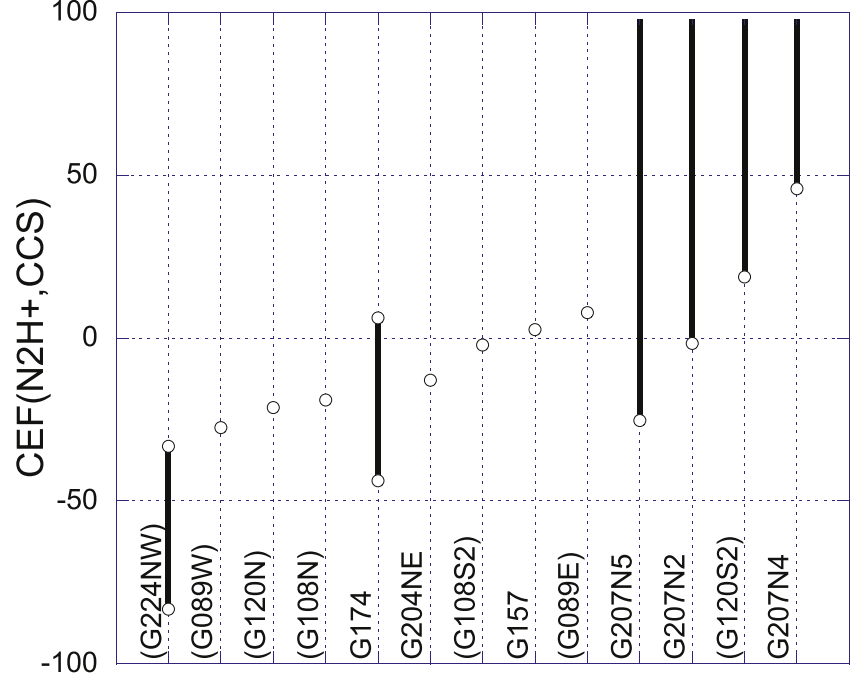

SCUBA-2 Peak

Figure 19. CEF for starless SCUBA-2 peaks based on the column density ratio of $N\left(\mathrm{~N}_{2} \mathrm{H}^{+}\right) / N(\mathrm{CCS})$. The source name in parentheses means distance is larger than $1 \mathrm{kpc}$. The range reflects the uncertainty for the $\mathrm{N}_{2} \mathrm{H}^{+}$optical depth for cores for which hyperfine fitting was not successful.

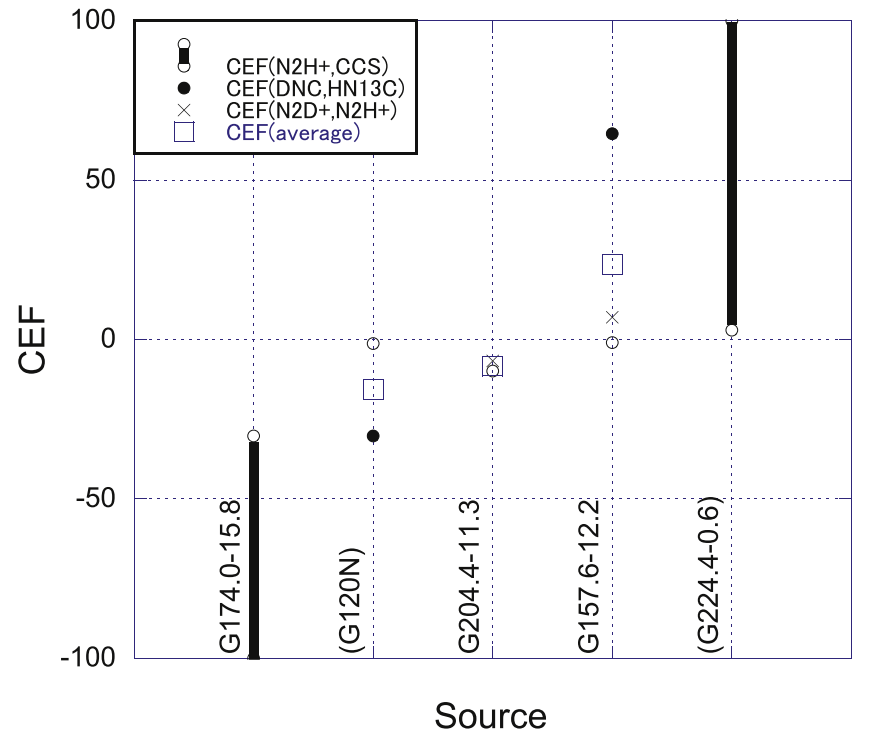

Figure 20. CEF at starless T70 positions based on multiple column density ratios. The source name in parentheses means distance is larger than $1 \mathrm{kpc}$. The range reflects the upper and lower limit to $N\left(\mathrm{~N}_{2} \mathrm{H}^{+}\right) / N(\mathrm{CCS})$.

inconsistency between CEF based on the deuterium fractionation and that based on $N\left(\mathrm{~N}_{2} \mathrm{H}^{+}\right) / N(\mathrm{CCS})$. $\mathrm{CEF}$ (average) does not include the upper or lower limit to $N\left(\mathrm{~N}_{2} \mathrm{H}^{+}\right) / N(\mathrm{CCS})$. For
$\mathrm{G} 108 \mathrm{~N}, \mathrm{CEF}\left(\mathrm{DNC}, \mathrm{HN}^{13} \mathrm{C}\right)$ is a lower limit, but because this is a starless core, we will not provide a positive CEF(DNC, $\left.\mathrm{HN}^{13} \mathrm{C}\right)$ value. That means $\mathrm{CEF}\left(\mathrm{DNC}, \mathrm{HN}^{13} \mathrm{C}\right)=-9$ to 0 nominally. However, this does not mean the estimate is very accurate. Then, we simply adopt this lower limit value of -9 to avoid being misleading. Figures 19 and 20 show that G174.015.8 is chemically young, and G204NE seems to be in the intermediate stage (on the verge of star formation).

The advantage of Planck cold clumps is that they are cold $(\lesssim 20 \mathrm{~K})$, and less affected by temperature effects. We have detected the $\mathrm{N}_{2} \mathrm{H}^{+}(1-0)$ emission from all the 13 clumps mapped with the Nobeyama $45 \mathrm{~m}$ telescope and receiver TZ1. The optically thin critical density for this transition is $6.1 \times 10^{4}$ and $4.1 \times 10^{4} \mathrm{~cm}^{-3}$ for 10 and $20 \mathrm{~K}$, respectively (Shirley 2015). The volume density $n$ for dense cores in cold molecular clouds detected in $\mathrm{N}_{2} \mathrm{H}^{+}(1-0)$ is $\gtrsim 3 \times 10^{4} \mathrm{~cm}^{-3}$ (Caselli et al. 2002). Thus, we can assume that the 13 mapped Planck cold clumps have densities higher than $\gtrsim 3 \times 10^{4} \mathrm{~cm}^{-3}$. With increasing density, the chemical reaction timescale will decrease. Then, the change in CEF will correspond to longer timescales for lower densities. The physical nature of the Planck cold clumps such as radius, mass, and volume density will be discussed in detail in a separate paper.

\subsection{Molecular Distribution}

Next, we investigate the morphology. In G089.9-01.9, the $82 \mathrm{GHz}$ and $94 \mathrm{GHz}$ CCS emission (young molecular gas) is distributed as if it surrounds the $\mathrm{N}_{2} \mathrm{H}^{+}$core (evolved gas). In G157.6-12.2, the $82 \mathrm{GHz}$ CCS emission is distributed as if it surrounds the $\mathrm{N}_{2} \mathrm{H}^{+}$core. Such configurations were previously reported in Aikawa et al. (2001) for L1544, and also starless $\mathrm{NH}_{3}$ core surrounded by CCS configurations are also observed by Lai \& Crutcher (2000) for L1498 and Tatematsu et al. (2014a) for Orion A GMC. L1544 shows evidence of the prestellar collapse. Therefore, these cores could be good targets for further studies for the initial conditions of star formation. For G157.6-12.2, CEF is $\gtrsim 0$, and its linewidth is as narrow as $0.3 \mathrm{~km} \mathrm{~s}^{-1}$. It is possible that this core is a coherent core that has largely dissipated turbulence, and is on the verge of star formation (Tatematsu et al. 2014a; Ohashi et al. 2016b). In G120S1, G120S2, and G224.4-0.6 $\mathrm{N}_{2} \mathrm{H}^{+}$and CCS distribution are largely different. G089.9-01.9, G120S, G157.6-12.2 are cold $(<11 \mathrm{~K})$, which may suggest depletion of CCS (cf., Aikawa et al. (2001), Bergin et al. (2002), Caselli et al. (2002). On the other hand, $\mathrm{N}_{2} \mathrm{H}^{+}$peaks are detected in the $\mathrm{HC}_{3} \mathrm{~N}$ emission in these cores. In $\mathrm{G} 149 \mathrm{~N}$, the $\mathrm{HC}_{3} \mathrm{~N}$ emission (young molecular gas) is distributed on the both sides of the $\mathrm{N}_{2} \mathrm{H}^{+}$core (evolved gas), which is also interesting. In G108N and G108S, the $82 \mathrm{GHz} \mathrm{GHz} \mathrm{CCS}$ and $\mathrm{N}_{2} \mathrm{H}^{+}$emission coexist roughly, but, 
again, this could be due to poorer physical resolution or a projection effect.

\section{Summary}

Thirteen Planck cold clumps were observed with the James Clerk Maxwell Telescope/SCUBA-2 and with the Nobeyama $45 \mathrm{~m}$ radio telescope. The $\mathrm{N}_{2} \mathrm{H}^{+}$spatial distribution is similar to SCUBA-2 dust distribution. The spatial distribution of $\mathrm{HC}_{3} \mathrm{~N}, 82 \mathrm{GHz} \mathrm{CCS}$, and $94 \mathrm{GHz} \mathrm{CCS}$ emission is often different from that of the $\mathrm{N}_{2} \mathrm{H}^{+}$emission. The CCS emission is often very clumpy. In G089.9-01.9 and G157.6-12.2, the CCS emission surrounds the $\mathrm{N}_{2} \mathrm{H}^{+}$core, which resembles the case of L1544 and suggests that they are on the verge of star formation. The detection rate of $\mathrm{N}_{2} \mathrm{D}^{+}$is $50 \%$. We investigated chemical evolutionary stages of starless Planck cold clumps using the newly defined CEF. We found that G174.0-15.8 is chemically young, and G089E, G157.6-12.2, and G204NE seem in the intermediate stage (on the verge of star formation). In addition, we observed $\mathrm{NH}_{3}$, and determined the kinetic temperature $T_{k}$.

M.K. was supported by the Basic Science Research Program through the National Research Foundation of Korea (NRF) funded by the Ministry of Science, ICT \& Future Planning (No. NRF-2015R1C1A1A01052160). M.J. acknowledges the support of the Academy of Finland Grant No. 285769. K.W. was supported by grant WA3628-1/1 of the German Research Foundation (DFG) through the priority program 1573 ("Physics of the Interstellar Medium").

We wish to thank the anonymous referee for the helpful comments. The James Clerk Maxwell Telescope is operated by the East Asian Observatory on behalf of The National Astronomical Observatory of Japan, Academia Sinica Institute of Astronomy and Astrophysics, the Korea Astronomy and Space Science Institute, the National Astronomical Observatories of China and the Chinese Academy of Sciences (Grant No. XDB09000000), with additional funding support from the Science and Technology Facilities Council of the United Kingdom and participating universities in the United Kingdom and Canada. The data analysis was carried out on the open-use data analysis computer system at the Astronomy Data Center, ADC, of the National Astronomical Observatory of Japan.

Facilities: JCMT, No:45m.

Software: AIPS, NewStar, NOSTAR.

\section{References}

Aikawa, Y., Ohashi, N., Inutsuka, S., Herbst, E., \& Takakuwa, S. 2001, ApJ, 552, 639

Asayama, S., \& Nakajima, T. 2013, PASP, 125, 213

Barnard, E. E. 1927, A Photographic Atlas of Selected Regions of the Milky Way ed. E. B. Frost \& M. R. Calvert (Washington, DC: Carnegie Institute of Washington)

Benson, P. J., Caselli, P., \& Myers, P. C. 1998, ApJ, 506, 743

Bergin, E. A., Alves, J., Huard, T. L., \& Tafalla, M. 2002, ApJL, 570, L101 Blitz, L. 1978, PhD thesis, Columbia Univ.

Bodenheimer, P. 1995, ARA\&A, 33, 199

Caselli, P., Benson, P. J., Myers, P. C., et al. 2002, ApJ, 572, 238

Caselli, P., \& Myers, P. C. 1995, ApJ, 446, 665

Chen, H.-R., Liu, S.-Yu., Su, Y.-N., \& Wang, M.-Y. 2011, ApJ, 743, 196 Clariá, J. J. 1974, AJ, 79, 1022

Crapsi, A., Caselli, P., Walmsley, C. M., et al. 2005, ApJ, 619, 379
Cummins, S. E., Linke, R. A., \& Thaddeus, P. 1986, ApJS, 60, 819

Dirienzo, W. J., Brogan, C., Indebetouw, R., et al. 2015, AJ, 150, 159 Dobashi, K., Uehata, H., Kandori, R., et al. 2005, PASJ, 57, 1

Emprechtinger, M., Wiedner, M. C., Simon, R., et al. 2009, A\&A, 496 731

Fontani, F., Caselli, P., Crapsi, A., et al. 2006, A\&A, 460, 709

Fontani, F., Sakai, T., Furuya, K., et al. 2014, MNRAS, 440, 448

Frerking, M. A., Langer, W. D., \& Wilson, R. W. 1979, ApJL, 232, L65

Geiss, J., \& Reeves, H. 1981, A\&A, 93, 189

Genzel, R., \& Stutzki, R. 1989, ARA\&A, 27, 41

Goldsmith, P. F., \& Arquilla, R. A. 1985, in Protostars and Planets II, ed

D. C. Black \& M. S. Mathews (Tucson, AZ: Univ. Arizona Press), 137

Goodman, A. A., Benson, P. J., Fuller, G. A., et al. 1993, ApJ, 406, 528

Hirahara, Y., Suzuki, H., Yamamoto, S., et al. 1992, ApJ, 394, 539

Hirota, T., Ikeda, M., \& Yamamoto, S. 2003, ApJ, 594, 859

Hirota, T., Ohishi, M., \& Yamamoto, S. 2009, ApJ, 699, 585

Hirota, T., \& Yamamoto, S. 2006, ApJ, 646, 258

Ho, P. T. P., \& Townes, C. H. 1983, ARA\&A, 21, 239

Hoq, S., Jackson, J. M., Foster, J. B., et al. 2013, ApJ, 777, 157

Kauffmann, J. 2007, PhD thesis, Univ. Bonn

Khavtassi, D. Sh. 1960, Atlas of Galactic Dark Nebulae (Abastumani: Abastumani Astrophysical Observatory)

Kim, B.-G., Kawamura, A., \& Fukui, Y. 1996, JKASS, 29, S193

Kim, M. K., Hirota, T., Honma, M., et al. 2008, PASJ, 60, 991

Kim, J., Lee, J.-E., Liu, T., et al. 2016, ApJ, submitted

Lai, S.-P., \& Crutcher, R. M. 2000, ApJS, 128, 271

Lee, J-. E., Bergin, E. A., \& Evans, N. J. 2004, ApJ, 617, 360

Liu, T., Wu, Y., Mardones, D., et al. 2015, PKAS, 30, 79

Liu, T., Wu, Y., \& Zhang, H. 2012, ApJS, 202, 4

Liu, T., Zhang, Q., Kim, K.-T., et al. 2016, ApJS, 222, 7

Loinard, L., Torres, R. M., Mioduszewski, A. J., et al. 2007, ApJ, 671, 546

Lombardi, M., Lada, C. J., \& Alves, J. 2010, A\&A, 512, 67

Lynds, B. T. 1962, ApJS, 7, 1

Maddalena, R. J., Morris, M., Moscowitz, J., et al. 1986, ApJ, 303, 375

Mangum, J. G., \& Shirley, Y. L. 2015, PASP, 127, 266

Nakajima, T., Kimura, K., Nishimura, A., et al. 2013, PASP, 125, 252

Nakano, M., Yoshida, S., \& Kogure, T. 1984, PASJ, 36, 517

Ohashi, S., Tatematsu, K., Choi, M., et al. 2014, PASJ, 66, 119

Ohashi, S., Tatematsu, K., Fujii, K., et al. 2016a, PASJ, 68, 3

Ohashi, S., Tatematsu, K., Sanhueza, P., et al. 2016b, MNRAS, 459, 413

Pickett, H. M., Poynter, R. L., Cohen, E. A., et al. 1998, JQRST, 60, 883

Planck Collaboration XXIII 2011, A\&A, 536, A23

Planck Collaboration XXVIII 2016, A\&A, 594, 28

Ramirez Alegria, S., Herrero, A., Mart in-Franch, A., et al. 2011, A\&A, 535, A8

Sakai, T., Sakai, N., Furuya, K., et al. 2012, ApJ, 747, 140

Sakai, T., Sakai, N., Furuya, K., et al. 2015, ApJ, 803, 70

Sakai, T., Sakai, N., Kamegai, K., et al. 2008, ApJ, 678, 1049

Sanhueza, P., Jackson, J. M., Foster, J. B., et al. 2012, ApJ, 756, 60

Sawada, T., Ikeda, N., Sunada, K., et al. 2008, PASJ, 60, 445

Schilke, P., Walmsley, C. M., Pineau des Forêts, G., et al. 1992, A\&A, 256, 595

Sharpless, S. 1959, ApJS, 4, 257

Shirley, Y. L. 2015, PASP, 127, 299

Snell, R. L., \& Wootten, A. 1979, ApJ, 228, 748

Suzuki, H., Yamamoto, S., Ohishi, M., et al. 1992, ApJ, 392, 551

Tafalla, M., Mardones, D., Myers, P. C., et al. 1998, ApJ, 504, 900

Tafalla, M., Myers, P. C., Caselli, P., et al. 2004, A\&A, 416, 191

Tatematsu, K., Fukui, Y., Nakano, M., et al. 1987, A\&A, 184, 279

Tatematsu, K., Hirota, T., Kandori, R., \& Umemoto, T. 2010, PASJ, 62, 1473

Tatematsu, K., Hirota, T., Ohashi, S., et al. 2014a, ApJ, 789, 83

Tatematsu, K., Nakano, M., Yoshida, S., et al. 1985, PASJ, 37, 345

Tatematsu, K., Ohashi, S., Sanhueza, P., et al. 2016, PASJ, 68, 24

Tatematsu, K., Ohashi, S., Umemoto, T., et al. 2014b, PASJ, 66, 16

Thaddeus, P., Guélin, M., \& Linke, R. A. 1981, ApJL, 246, L41

Tiné, S., Roueff, E., Falgarone, E., Gerin, M., \& Pineau des Forêts, G. 2000, A\&A, 356, 1039

Wootten, A. 1987, in IAU Symp. 120, Astrochemistry, ed. M. S. vardya \& S. P. Tarafdar (Dordrecht: Reidel), 311

Wouterloot, J. G. A., \& Habing, H. J. 1985, A\&AS, 60, 43

Wu, Y., Liu, T., Meng, F., et al. 2012, ApJ, 756, 76

Yamamoto, S., Saito, S., Kawaguchi, K., et al. 1990, ApJ, 361, 318 